\title{
Skin-Derived Precursors Generate Myelinating Schwann Cells That Promote Remyelination and Functional Recovery after Contusion Spinal Cord Injury
}

\author{
Jeff Biernaskie, ${ }^{1 \star}$ Joseph S. Sparling, ${ }^{4,5 \star}$ Jie Liu, ${ }^{4,5}$ Casey P. Shannon, ${ }^{4,5}$ Jason R. Plemel, ${ }^{4,5}$ Yuanyun Xie, ${ }^{4,5}$ \\ Freda D. Miller, ${ }^{1,2,3}$ and Wolfram Tetzlaff $f^{4,5,6}$ \\ ${ }^{1}$ Developmental and Stem Cell Biology Group, Hospital for Sick Children, and Departments of ${ }^{2}$ Molecular and Medical Genetics and ${ }^{3}$ Physiology, University \\ of Toronto, Toronto, Ontario, Canada M5G 1L7, and ${ }^{4}$ International Collaboration on Repair Discoveries, and Departments of ${ }^{5}$ Zoology and ${ }^{6}$ Surgery, \\ University of British Columbia, Vancouver, British Columbia, Canada V6T 1 Z4
}

Transplantation of exogenous cells is one approach to spinal cord repair that could potentially enhance the growth and myelination of endogenous axons. Here, we asked whether skin-derived precursors (SKPs), a neural crest-like precursor that can be isolated and expanded from mammalian skin, could be used to repair the injured rat spinal cord. To ask this question, we isolated and expanded genetically tagged murine SKPs and either transplanted them directly into the contused rat spinal cord or differentiated them into Schwann cells (SCs), and performed similar transplantations with the isolated, expanded SKP-derived SCs. Neuroanatomical analysis of these transplants 12 weeks after transplantation revealed that both cell types survived well within the injured spinal cord, reduced the size of the contusion cavity, myelinated endogenous host axons, and recruited endogenous SCs into the injured cord. However, SKP-derived SCs also provided a bridge across the lesion site, increased the size of the spared tissue rim, myelinated spared axons within the tissue rim, reduced reactive gliosis, and provided an environment that was highly conducive to axonal growth. Importantly, SKP-derived SCs provided enhanced locomotor recovery relative to both SKPs and forebrain subventricular zone neurospheres, and had no impact on mechanical or heat sensitivity thresholds. Thus, SKP-derived SCs provide an accessible, potentially autologous source of cells for transplantation into and treatment of the injured spinal cord.

Key words: skin-derived precursors; Schwann cells; spinal cord; transplantation; myelination; stem cells; recovery of function; neural stem cells; axonal growth; axonal regeneration

\section{Introduction}

After injury, the adult mammalian spinal cord exhibits limited endogenous repair and poor functional recovery. Several factors contribute to this, including protracted secondary damage, an inability to remyelinate spared demyelinated axons, and failure of axons to overcome local expression of myelin-associated inhibitory molecules and the reactive astrocytic scar (Deumens et al., 2005; Dietz and Curt, 2006; Harel and Strittmatter, 2006; Yiu and $\mathrm{He}, 2006)$. The early pioneering work of Aguayo and colleagues

Received Nov. 22, 2006; revised June 10, 2007; accepted June 13, 2007.

This work was supported by a grant from NeuroScience Canada. J.B. was supported by fellowships from the Parkinsons Society of Canada and the Canadian Institutes of Health Research (CIHR). J.S.S. was supported by a CIHR Transplant Research Training Award. J.R.P. was supported by a Michael Smith Foundation for Health Research Studentship. F.D.M. is a Canada Research Chair and a Howard Hughes Medical Institute International Research Scholar. W.T. holds the Edie Ehlers Chair in Spinal Cord Injury Research (Rick Hansen Foundation). The technical assistance of Darren Sutherland, Clarrie Lam, Jae Lee, Angel Wong, and Anne Aumont is gratefully acknowledged.

*J.B. and J.S.S. contributed equally to this work.

Correspondence should be addressed to either of the following: Dr. Freda D. Miller, Hospital for Sick Children MaRS Centre, Toronto Medical Discovery East Tower, 101 College Street, 12th Floor, Room 12-313, Toronto, Ontario, Canada M5G 1L7, E-mail: fredam@sickkids.ca; or Dr. Wolfram Tetzlaff, Biological Sciences Building, Zoology/lnternational Collaboration on Repair Discoveries Room 2469, University of British Columbia, 6270 University Drive, Vancouver, British Columbia, Canada V6T 1Z4, E-mail: tetzlaff@icord.org.

DOI:10.1523/JNEUROSCI.1930-07.2007

Copyright $\odot 2007$ Society for Neuroscience $\quad 0270-6474 / 07 / 279545-15 \$ 15.00 / 0$
(Richardson et al., 1980; David and Aguayo, 1981) demonstrated that the peripheral nerve environment was conducive for CNS axon regeneration, and subsequent work demonstrated that Schwann cells (SCs) comprised an essential component of this permissive environment. More recently, numerous studies have demonstrated that transplantation of exogenous peripheral nerve SCs can provide both trophic support for spared axons and participate in remyelination of the injured spinal cord (Xu et al., 1997; Keirstead et al., 1999; Kocsis et al., 2002; Bunge and Pearse, 2003; Pearse et al., 2004a; Oudega and Xu, 2006; Pearse and Barakat, 2006). However, the invasive surgical biopsies required to harvest the nerve, and the difficulties faced in the purification and expansion of SCs from adult nerves (Akiyama et al., 2002), have complicated their clinical application.

Skin-derived precursors (SKPs) are a self-renewing, multipotent precursor that resides within the dermis of both rodents and humans (Toma et al., 2001, 2005). SKPs, which are generated during embryogenesis and persist into adulthood, share characteristics with embryonic neural crest stem cells (Fernandes et al., 2004), including their ability to differentiate into neural crestderived cell types such as peripheral neurons and SCs (Fernandes et al., 2004, 2006; McKenzie et al., 2006). In this regard, we recently demonstrated that SKPs respond to neural crest cues such 
as neuregulins by generating SCs, and that these SKP-derived SCs (SKP-SCs) express a myelinating phenotype when cocultured with peripheral axons. Moreover, SKP-SCs display similar behavior in vivo, generating compact myelin when they encounter axons either within the regenerating peripheral nerve or within the dysmyelinated CNS (McKenzie et al., 2006). Because SKPs represent a highly accessible and potentially autologous source of adult precursors that are capable of generating functional SCs, we asked whether naive SKPs or SKP-SCs could be used to repair the injured spinal cord by remyelinating spared axons, and/or by providing a growth-permissive environment for the regeneration of damaged axons. As a transplant control, we compared naive SKPs and SKP-SCs to neurospheres derived from the forebrain subventricular zone (SVZ), a well characterized CNS stem cell that is capable of generating oligodendrocytes and that has been reported to provide some benefit after spinal cord injury (Ogawa et al., 2002; Okano et al., 2003; Vroemen et al., 2003; Watanabe et al., 2004; Karimi-Abdolrezaee et al., 2006). Here, we report that SKP-SCs provide a tissue bridge across the site of a contusion lesion, myelinate host axons both in the spared tissue rim and within the transplant site itself, modify the extracellular milieu around the lesion, and ultimately lead to enhanced functional locomotor recovery after spinal cord injury.

\section{Materials and Methods \\ Animals}

Sixty-two adult male Sprague Dawley rats were used in this study. All procedures were approved by the Hospital for Sick Children Research Institute and the University of British Columbia, in accordance with guidelines of the Canadian Council on Animal Care.

\section{Preparation of SKPs and SKP-SCs}

Neonatal murine SKPs were cultured as described previously (Toma et al., 2001; Fernandes et al., 2004). Briefly, dorsal back skin from neonatal [postnatal day 0 (P0) to P3] enhanced yellow fluorescent protein (EYFP) mice (The Jackson Laboratory, Bar Harbor, ME) was cut into $2-3 \mathrm{~mm}^{2}$ pieces. Tissue was digested with $1 \mathrm{mg} / \mathrm{ml}$ collagenase (type XI; Sigma, St. Louis, $\mathrm{MO}$ ) for $20-45 \mathrm{~min}$ at $37^{\circ} \mathrm{C}$, mechanically dissociated and filtered through a $40 \mu \mathrm{m}$ cell strainer (BD Falcon, Bedford, MA). Cells were plated in DMEM/F-12 (3:1; Invitrogen, Carlsbad, CA), containing 1\% penicillin/streptomycin (Cambrex, East Rutherford, NJ), 2\% B27 supplement (Invitrogen), $20 \mathrm{ng} / \mathrm{ml}$ epidermal growth factor (EGF), and 40 $\mathrm{ng} / \mathrm{ml}$ fibroblast growth factor-2 (FGF2) (both Collaborative Research, Bedford, MA). To generate secondary spheres, SKPs were digested in collagenase $(1 \mathrm{mg} / \mathrm{ml})$ for $5 \mathrm{~min}$ and mechanically dissociated to single cells and then subcultured at a density of $10-25,000$ cells $/ \mathrm{ml}$ to generate secondary spheres.

Differentiation of SKPs to SCs (SKP-SCs) was done as previously described (Biernaskie et al., 2006; McKenzie et al., 2006). Briefly, primary or secondary spheres were dissociated and plated on $10 \mathrm{~cm}$ dishes coated with poly-D-lysine/laminin (BD Biosciences, Bedford, MA), and grown in DMEM/F-12 (3:1) containing $5 \mu \mathrm{M}$ forskolin (Sigma) and $50 \mathrm{ng} / \mathrm{ml}$ neuregulin-1 $\beta$ (R\&D Systems, Minneapolis, MN) and supplemented with $1 \% \mathrm{~N} 2$ (Invitrogen). The medium was changed every 3-4 d. Proliferating colonies of SKP-SCs were isolated using cloning cylinders (Corning, Corning, NY), trypsinized from the dish, and replated in the same medium. Cultures of increasing purity were obtained by sequential passaging as cultures reached confluency.

Neonatal neurospheres were generated from the forebrain subventricular zone of the same postnatal EYFP mice that were used for the SKP cultures, and were used as controls for the cell transplantation studies. Specifically, after removal of the back skin, brains of the same mice were carefully dissected to isolate the tissue surrounding the forebrain lateral ventricles. The tissue was briefly digested with $0.1 \%$ trypsin, gently dissociated to single cells by trituration, and grown at a concentration of 10,000 cells/ml. Neurospheres were grown under identical conditions to those used for SKPs (DMEM/F-12 medium containing FGF2, EGF, and
B27). Floating spheres appeared after $5 \mathrm{~d}$ and were passaged every $5 \mathrm{~d}$ using trypsin digestion and mechanical dissociation. All neurospheres underwent at least one, and no more than three, passages before transplantation.

\section{Flow cytometry}

Before transplantation, flow cytometry was used to assess the percentage of cells staining with the p75 neurotrophin receptor, a marker of SC fate, after differentiation and expansion of SKPs. We also examined the percentage of SKP-SCs maintaining expression of yellow fluorescent protein (YFP). SKP-SC were isolated using cloning cylinders and expanded for 6 weeks in SC differentiation conditions as described previously (McKenzie et al., 2006). SKP-SCs were trypsinized using trypsin-versene (BioWhittaker, Walkersville, MD) and resuspended in HBSS containing 10\% FBS to inactivate the enzyme. Cells were centrifuged at $1200 \mathrm{rpm}$ for 6 min and resuspended in $4 \%$ paraformaldehyde in $0.1 \mathrm{~m}$ phosphate buffer for $15 \mathrm{~min}$. Next, cells were washed twice by centrifuging at $1200 \mathrm{rpm}$ for 6 min and resuspension in PBS containing 1\% bovine serum albumin (wash buffer). For staining, cells were resuspended with a polyclonal rabbit anti-p75 neurotrophin receptor (p75NTR) (intracellular domain, 1:1000; Promega, Madison, WI) PBS containing 0.3\% Triton X-100 and $1 \%$ normal goat serum for $30 \mathrm{~min}$. Cells were again washed twice by centrifuging at $1200 \mathrm{rpm}$ for $6 \mathrm{~min}$ and resuspended in wash buffer. Staining was visualized using an Alexa 647-conjugated anti-rabbit secondary antibody (1:2000; Invitrogen) in PBS containing 1\% BSA. For negative controls, exposure to the primary antibody was omitted. Analysis was done using a FACSCalibur flow cytometer (BD Biosciences), and 10,000 events were collected for each population.

\section{Spinal cord contusion injury and cell transplantation}

Adult male Sprague Dawley rats (286 \pm 7 g; Charles River Laboratories, Wilmington, MA) were acclimatized to the behavioral testing environment (see below) to establish their behavioral baselines. All surgeries were performed under aseptic conditions. Body temperature was maintained at $>36^{\circ} \mathrm{C}$ throughout the surgical and postsurgical period, and blood oxygenation level and heart rate were monitored. Rats were anesthetized with a mixture of ketamine hydrochloride $(72 \mathrm{mg} / \mathrm{kg})$ and xylazine hydrochloride $(9 \mathrm{mg} / \mathrm{kg})$ via intraperitoneal injection. The skin of the back was shaved and disinfected, and the dorsal aspect of the spinal column exposed at the T8-T10 vertebrae. A laminectomy of vertebra T9 was performed using a fine pair of rongeurs and care was taken not to damage the dura. Rats were then transferred to the stage of the Ohio State University spinal cord impactor device and the dorsal process of vertebrae T8 and T10 held in place with Allen clamps while $50 \%$ of the body weight was supported from underneath. The initial touch point of the impactor with the dura was determined (using the vibrator mode of the impactor tip), and from there a $1.5 \mathrm{~mm}$ displacement with $6 \mathrm{~ms}$ dwell time was applied to the spinal cord. Force curve readings revealed values with an average of $286 \pm 5 \mathrm{kdyn}$. Slippage or excessive deviation from these values led to automatic exclusion of the animal from additional study ( $\sim 15 \%$ in our hands). This type of injury consistently results, in our hands, in complete loss of hindlimb motor function on day 2 [Basso, Beattie, Bresnahan (BBB) score $<2$ ], followed by gradual recovery of hindlimb weight supported stepping with no or only occasional forelim$\mathrm{b}$-hindlimb coordination ( $\mathrm{BBB} \approx 11$ ). Immediately after the injury and for an additional day, rats received Ringer's solution for hydration $(5 \mathrm{ml}$, s.c.) and buprenorphine $(0.3 \mathrm{mg} / \mathrm{kg})$ to alleviate pain. Bladders were expressed three times daily until the rats reached spontaneous micturition. Antibiotics (Baytril; $0.06 \mathrm{ml}$, i.m.) were given to treat/prevent bladder infection.

For transplantations, $7 \mathrm{~d}$ after contusion injury, rats received no treatment (medium injection alone, $n=4$ ) or were transplanted with one of three different cell types: neonatal murine SVZ neurospheres $(n=11)$, undifferentiated neonatal SKPs $(n=13)$, or SKP-SCs $(n=16)$. Immediately before transplantation, spheres of SKPs or neurospheres were digested with $1 \mathrm{mg} / \mathrm{ml}$ collagenase and gently triturated to yield a singlecell suspension. Cells were resuspended at a final concentration of $2 \times$ $10^{5}$ cells $/ \mu \mathrm{l}$, and a total volume of $5 \mu \mathrm{l}\left(8 \times 10^{5}\right.$ total cells $)$ was stereotaxically injected into the epicenter of the lesion using a $10 \mu \mathrm{l} \mathrm{Hamil-}$ 
ton syringe fitted with a glass micropipette. All animals received daily subcutaneous injections of cyclosporine A ( $15 \mathrm{mg} / \mathrm{kg}$, s.c.; Novartis, East Hanover, NJ) beginning $2 \mathrm{~d}$ before transplantation and continuing for the duration of the experiment. Behavioral testing continued for 8 weeks after transplantation, and the animals were reanesthetized at 10 weeks after injury (as described above) to trace the ascending sensory fibers and descending corticospinal fibers. To trace sensory fibers, cholera toxin B (CTB) $(1 \mu \mathrm{l}$; List Laboratories, Campbell, CA) was injected into the lumbar sciatic nerve at the sciatic notch. Alternatively, to trace the corticospinal tract, biotinylated dextrane amine (BDA) (10\%; four times 0.5 $\mu \mathrm{l}$; Invitrogen) was injected into the hindlimb area of sensorimotor cortex using a fine glass capillary attached to a Hamilton syringe. Injection was controlled by an automatic advancement device, which allowed the tracer to be injected at rates not exceeding $100 \mathrm{nl} / \mathrm{min}$. For ethical reasons, rats were randomized to receive either CTB or BDA, but not both. Neuroanatomical tracing procedures precluded additional behavioral testing of the animals.

To quantitatively assess the effect of transplant on axonal growth, a subset of animals were engrafted with either SKP-SCs $(n=10)$ or medium only $(n=8)$ and were killed at 1 or 2 weeks after transplantation. All other rats were killed 1-2 weeks after the tracer injections ( $\sim 12$ weeks after injury) with a lethal dose of ketamine/xylazine and were then transcardially perfused with PBS, followed by $4 \%$ paraformaldehyde. The spinal cords were dissected, postfixed overnight in the same fixative, and cryoprotected with $30 \%$ sucrose in $0.1 \mathrm{M}$ PBS.

\section{Tissue processing and immunocytochemistry}

Immunocytochemistry on cultured cells was performed in plastic chamber slides or dishes as we described previously (McKenzie et al., 2006). For spinal cords, $20 \mu \mathrm{m}$ longitudinal sections were cut on a cryostat in the horizontal plane and mounted on Superfrost Plus slides (Fisher, Houston, TX). Sections were permeabilized with $0.2 \%$ Triton X-100, and nonspecific binding sites were subsequently blocked with $10 \%$ normal goat or donkey serum. For immunodetection of myelin basic protein (MBP) and $\mathrm{P}_{0}$, tissue sections were delipidized before any staining procedures. Primary antibodies were incubated overnight at $4^{\circ} \mathrm{C}$, washed, and then incubated with fluorescent-conjugated secondary antibodies for $2 \mathrm{~h}$ at room temperature. The following primary antibodies were used: anti- $\beta$ III-tubulin monoclonal (1:500; Covance, Princeton, NJ), rabbit anti-neurofilament $\mathrm{M}$ (NFM) (1:500; Chemicon, Temecula, $\mathrm{CA})$, polyclonal chicken anti-green fluorescent protein (1:1000; Chemicon), polyclonal chicken anti- $\mathrm{P}_{0}(1: 1000$; Aves Labs, Tigard, OR), antiGFAP polyclonal (1:500; DakoCytomation, Carpinteria, CA), polyclonal anti-p75NTR (1:500; Promega or Chemicon), monoclonal anti-rat p75NTR (1:500; Chemicon), anti-S100 $\beta$ monoclonal (1:1000; Sigma), polyclonal anti-collagen type II (1:200; Chemicon), polyclonal antifibronectin (1:400; Sigma), monoclonal anti-neurocan (1:500; Chemicon), monoclonal anti-cholera toxin B (1:500; Chemicon), anti-MBP monoclonal (1:200; Chemicon), anti-MBP monoclonal (1:100; Serotec, Oxford, UK), rabbit anti-laminin (1:200; Sigma), polyclonal antityrosine hydroxylase (TH) (1:200; Chemicon), rabbit anti-serotonin transporter (1:500; Immunostar, Hudson, WI), rabbit anti-calcitonin gene-related peptide (CGRP) (1:500; Sigma), polyclonal anti-NG2 (1: 200; Chemicon), anti-Ki67 monoclonal (1:200; BD Pharmingen, San Diego, CA), polyclonal anti-contactin-associated protein (Caspr; a generous gift from Dr. J. Trimmer, University of California, Davis, CA), and anti-Kv1.2 potassium channels (Dr. J. Trimmer). Secondary antibodies used were as follows: Alexa 488-conjugated goat anti-mouse, Alexa 555 goat anti-mouse IgM, Alexa 555 goat anti-rabbit, and Alexa 350 goat anti-mouse (1:1000; all from Invitrogen) and FITC-conjugated, AMCA (7-amino-4-methylcoumarin-3-acetic acid)-conjugated, or Cy3conjugated donkey anti-mouse, -rabbit, -chick, or -goat (Jackson ImmunoResearch, West Grove, PA). Immunofluorescence was visualized using a Zeiss (Oberkochen, Germany) Axioplan 2 microscope fitted with deconvolution software (Northern Eclipse; Empix, Mississauga, Ontario, Canada). Colocalization was confirmed by adjacent $1 \mu \mathrm{m}$ optical sections using a Zeiss LSM 5 confocal laser-scanning microscope.
Quantification of cavity and transplant volume, tissue sparing, cell survival, myelination, and axon numbers

To determine transplant volumes, serial spinal cord sections $(200 \mu \mathrm{m}$ apart) immunostained for YFP were photographed at $10 \times$ primary magnification. Using Northern Eclipse software (Empix), regions containing YFP-expressing cells were outlined and the area was measured. Transplant volumes were determined using the following equation: $V=$ $\Sigma$ [area $X$ section thickness $X$ number of sections in each sampling block]. Quantification of cavity volumes were determined in an identical manner, using serial photographs of YFP and $\mathrm{P}_{0}$ immunofluorescence. To estimate the amount of spared spinal cord tissue, widths of the thinnest portion of spared tissue around the lesion cavity (i.e., spared host rim) were measured every $200 \mu \mathrm{m}$ through the extent of each spinal cord. These measurements were then summed, providing a conservative estimate of spared host tissue for each animal.

Survival of transplanted, YFP-positive cells was determined for the five animals with the largest transplant volumes in each group using unbiased stereological techniques. The spinal cord was divided longitudinally into three blocks. One section from each block was sampled such that three randomly selected regions of the transplant from each section were photographed at $40 \times$ primary magnification. The number of YFP-positive cells as well as the number of YFP-positive cells generating a $\mathrm{P}_{0}$-positive myelin sheath within each given area were determined, and the mean density $\left(\right.$ cells $/ \mathrm{mm}^{3}$ ) for each block was calculated. The total number of surviving YFP-positive cells was then estimated by multiplying by the transplant volume.

To quantify endogenous SC remyelination, the spinal cord was again divided into three blocks. One spinal cord section from each block was sampled to determine all of the $\mathrm{P}_{0}$-positive myelin sheaths within the lesion in that section versus those within the intact host tissue in the same section. The total number of $\mathrm{P}_{0}$-positive myelin sheaths within each block was calculated using the following equation: number of $\mathrm{P}_{0}$ sheaths $=\Sigma$ [number of cells $\times$ number of sections in each block].

To determine the effect of transplantation on axonal sparing, or sprouting/growth, the number of axon fibers found within the lesion at 1 , 2 (medium control vs SKP-SC), and 11 weeks (SKP-SCs vs SKPs vs neurospheres) after transplant were counted. Spinal cords were immunostained for TH or 5-hydroxytryptamine (5-HT) to identify a subset of descending axon fibers, and these fibers were counted at $200 \times$ magnification. Lesion cavity was defined as the areas of tissue containing transplanted (YFP-positive) cells and/or regions within the lesion cavity that exhibited a loss of GFAP-positive astrocytes (typical of intact host CNS tissue). This is an important consideration particularly with regard to medium-treated control animals who, during the first 2 weeks after injury, contain areas rich with immune cells (i.e., macrophages) but few astrocytes, and yet still contain axon fibers that are presumably spared or sprouting. From $20 \mu \mathrm{m}$ longitudinal sections, every 10th section through the entire thickness of the spinal cord was sampled. Data are presented as group means of actual counts $( \pm S E M)$ and have not been normalized for the entire cord. Five animals from each group were examined at each time point, with the exception of the 1 week medium control group $(n=$ 3). All measurements and counts were done by an observer who was blinded to experimental conditions.

\section{Behavioral assessment}

Behavioral assessments of locomotor and sensory function were conducted to compare the functional outcome of various treatments over time. Hindlimb locomotor function was assessed by open-field locomotion and horizontal-ladder paradigms, whereas hindlimb sensory function was assessed using sensitivity tests of mechanical or thermal stimuli. All behavioral tests were conducted 1-2 d before injury to establish baseline values. Open-field testing was done again at $6 \mathrm{~d}$ after injury, and the resulting $\mathrm{BBB}$ score was used to eliminate animals that demonstrated a score $>10$ at that time point to standardize injury severity. The animals were transplanted at $7 \mathrm{~d}$ after contusion and were then allowed to rest for 1 week before resuming behavioral testing. Open-field testing was conducted on a weekly basis from 2 weeks after injury (i.e., 1 week after transplant) to 9 weeks after injury. All other behavioral tests were delayed until the animals had regained plantar placement with weight support. 
Specifically, the horizontal-ladder test was conducted again at 5 and 9 weeks after injury, whereas the sensory tests were conducted at 5, 7, and 9 weeks after injury.

Open-field test. For open-field locomotion testing, the animals were placed in a large Plexiglas open field and observed by two raters (blinded to treatment) for $4 \mathrm{~min}$ to assess their performance using the BBB locomotor rating scale (Basso et al., 1995) and the BBB subscore scale (Basso, 2004). Briefly, the BBB is a scale from 0 to 21 that provides a gross indication of locomotor ability. Scores from 0 to 8 indicate improving degrees of movement of hip, knee, and ankle joints, whereas scores from 9 to 14 indicate various stages of weight support, stepping, and coordination. In our hands, medium-treated animals with moderate contusion injuries such as those used in this experiment commonly plateau at consistent weight-supported stepping with no forelimb-hindlimb coordination (BBB, 11) between 5 and 12 weeks after injury (data from previous work not shown). This scale fails to account for finer details of locomotion (toe clearance, paw position, and tail position, which impact BBB scores from 15 to 21) until a rat has achieved consistent coordination of weight supported plantar stepping $(\mathrm{BBB}, 14)$. As such, we used the BBB subscore to assess individual components of hindlimb locomotor function, separate from the overall BBB score. The subscore is a scale ranging from 0 to 13 , with 0 representing no parallel paw positioning, no toe clearance, and no tail lifts, and 13 representing parallel paw positioning at initial contact and liftoff, consistent toe clearance, and a raised tail during locomotion. All animals included in the present study scored a 21 on the $\mathrm{BBB}$ and $\mathrm{a} 13$ on the $\mathrm{BBB}$ subscore during baseline assessments. The cage mates that had already completed testing on a given day were occasionally placed in the open field to promote spontaneous locomotion of less cooperative animals, and animals were occasionally given an extra minute in the open field to ensure adequate movement for accurate scoring.

Horizontal-ladder test. Error (i.e., footslip) frequency was assessed using a horizontal ladder modified as described previously (Metz and Whishaw, 2002). Briefly, rats were videotaped as they walked along a horizontal ladder with variable rung spacing. Subsequent video analysis provided scores for number of steps overall and number of errors (i.e., missteps or footslips) and the total number of errors was divided by the total number of steps to provide an error ratio that was used as the final measure of functional outcome.

Mechanical sensitivity test. The threshold of mechanical stimulation for each rat was tested using a Dynamic Plantar Aesthesiometer (Ugo Basile, Comerio, Italy). Similar to an automated von Frey hair test, this device pushes a thin, semirigid, plastic probe through a wire-grated floor and against the plantar surface of the paw from beneath. The probe was set to exert a load increasing at a rate of $5 \mathrm{~g} / \mathrm{s}$ to a maximum load of $50 \mathrm{~g}$ and the device automatically stops and records the force at which the animal withdraws the limb. Each hindlimb was tested twice and the maximum force applied before withdrawal was averaged for all four values as a measure of that animal's threshold of response to mechanical stimulation in a given testing week. Those values were compared with baseline measurements acquired before injury to determine whether any of the treatments elicited responses indicative of mechanical allodynia (i.e., neuropathic pain).

Thermal sensitivity test. This test used an infrared (IR) plantar test device (Ugo Basile) based on the Hargreaves method (Hargreaves et al., 1988) to assess latency of withdrawal after exposing the hindlimb plantar surface to a radiant heat source. The IR intensity of the device was set at $50 \%$ to ensure that the animals would suffer no tissue damage even at the maximum duration of heat application (set at $30 \mathrm{~s}$ ). The device shuts off automatically and records the latency when an animal withdraws it foot from the IR beam. On testing days, latency of withdrawal from the IR source was assessed twice for each hindlimb, for a total of four measures, which were averaged to provide the threshold of response to heat stimulation each week. Those values were compared with baseline to determine whether any of the treatments caused thermal hyperalgesia indicative of neuropathic pain in response to heat stimulation.

\section{Statistical analyses}

All data are presented as mean \pm SEM. Histological data, including cavity volume, cell survival, myelination, and axon counts, were analyzed using one-way ANOVA, and group differences were ascertained using Fisher's PLSD post hoc comparisons. Behavioral data were analyzed using repeated-measures ANOVA, and group differences were ascertained using unpaired $t$ tests. For data that violated the assumptions of normality and/or homogeneity of variance (i.e., von Frey mechanical sensitivity test) of the parametric tests, the equivalent nonparametric tests (Kruskal-Wallis and Mann-Whitney $U$ ) were used. The significance level for all tests was set at $p<0.05$.

\section{Results}

Isolation, expansion, and characterization of SKPs and SKPderived SCs for transplantation

We previously showed that SKPs will efficiently generate myelinating SCs when differentiated in the presence of neuregulins (McKenzie et al., 2006). Moreover, even naive SKPs will generate myelinating SCs when transplanted into the regenerating peripheral nerve (McKenzie et al., 2006). Because SCs have been proposed as a treatment for spinal cord injury, we generated both SKPs and SKP-SCs for transplantation experiments. Specifically, YFP-tagged SKPs were generated from neonatal (postnatal days 1-3) mouse backskin by culturing in FGF2 and EGF and passaging the floating spheres at 2 weeks, as we described previously (Toma et al., 2001; Fernandes et al., 2004; Biernaskie et al., 2006; McKenzie et al., 2006). These secondary SKP spheres expressed characteristic markers, including nestin, fibronectin, and versican (Fernandes et al., 2004) and were either used directly for transplantation, or were differentiated into SKP-SCs before transplantation. For these differentiations, SKPs were cultured in the presence of neuregulin-1 $\beta$ and forskolin (Biernaskie et al., 2006; McKenzie et al., 2006), SKP-SCs were identified morphologically, mechanically isolated, and then further expanded in the same gliogenic conditions. After three passages, the putative SKP-SCs had expanded significantly, maintaining both their bipolar morphology (Fig. 1A) and their expression of the YFP reporter gene (Fig. $1 B$ ). The purity of these SKP-SC cultures was confirmed by flow cytometry, which demonstrated that $\geq 97 \%$ of the cells were positive for both the YFP reporter, and for p75NTR (Fig. 1C,D), a marker of SCs or neural crest precursors. Moreover, immunostaining confirmed that the YFP-positive SKP-SCs coexpressed $\mathrm{S} 100 \beta$, p75NTR, and $\mathrm{P}_{0}$ (Fig. $1 E, F$ ), an expression profile characteristic of SCs and SC precursors.

As a control for these transplantations, we generated neurospheres from the neonatal forebrain SVZ, because SVZ-derived neurospheres have previously been transplanted into the injured rat spinal cord (Vroemen et al., 2003; Karimi-Abdolrezaee et al., 2006). To obtain these cells, we used brains from the same neonatal, YFP-expressing mice used to generate SKPs. We previously characterized similar cultures and demonstrated that they express appropriate markers and differentiate into neurons, astrocytes, and oligodendrocytes (Reynolds and Weiss, 1992; Reynolds et al., 1992; Morshead et al., 1994; Craig et al., 1996).

\section{Transplanted SKPs and SKP-derived SCs survive in the contusion lesion cavity and SKP-derived SCs promote sparing of the host spinal cord tissue}

To ask whether SKPs or SKP-SCs represent a potential cell source for treatment of spinal cord injury, we contused rats and then transplanted cells into the epicenter of the spinal cord lesion cavity 1 week later. Animals were then assessed for sensorimotor function over 9 weeks and examined neuroanatomically at 12 weeks after injury. 


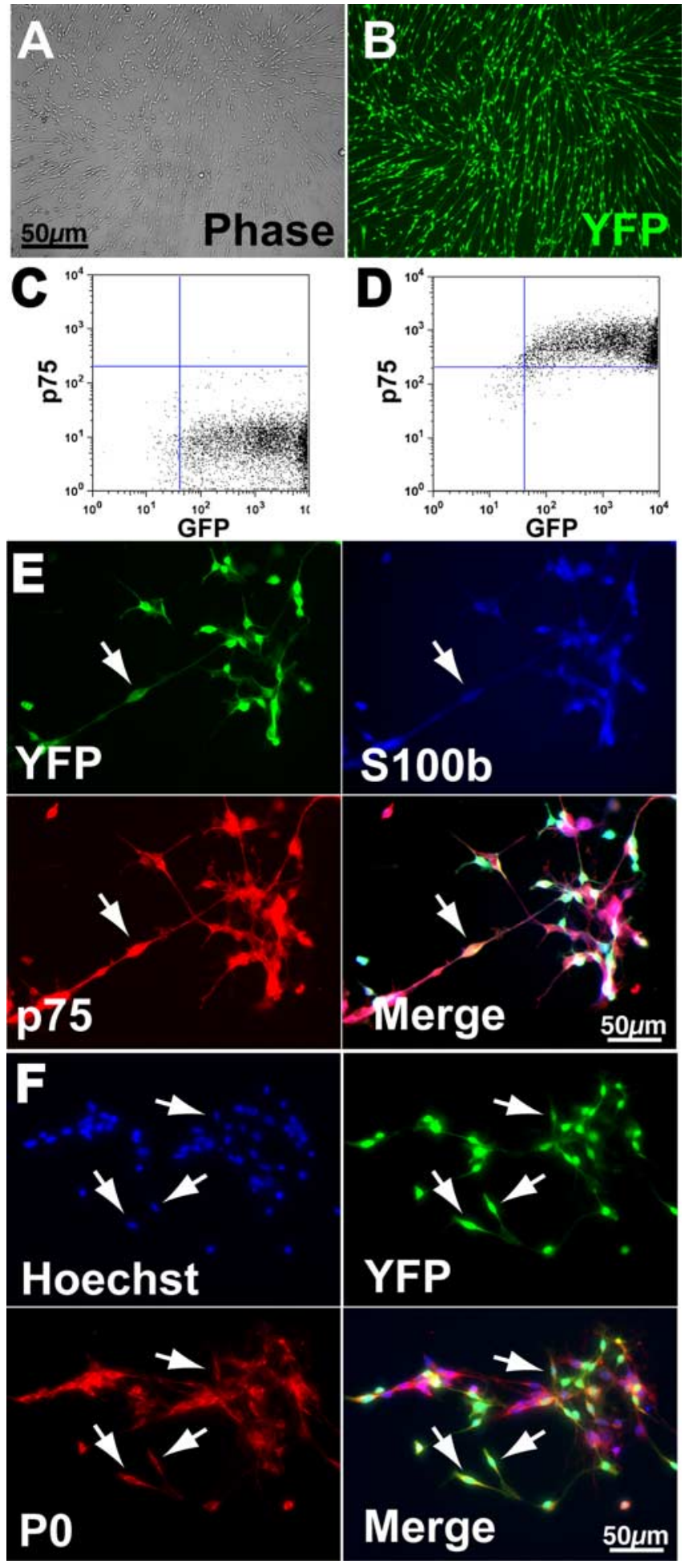

Figure 1. Characterization of SKP-derived SCS before transplantation. $A, B$, Photomicrographs of SKP-derived SCs generated from a mouse that expresses YFP in all cell types. Both panels show the same field photographed with phase illumination $(\boldsymbol{A})$ and with fluorescence illumination $(\boldsymbol{B})$. C , D, Flow cytometry with SKP-derived SCs for the genetic YFP tag $(\boldsymbol{C} ;$ GFP) and for p75NTR ( $\boldsymbol{D} ; \mathrm{p} 75)$, a cell surface marker for SCs. Note that $>97 \%$ of SKP-derived SCs express both of these proteins. $\boldsymbol{E}$, Photomicrographs of SKP-derived SCs triple-labeled for the genetic YFP tag, and the SC markers S100 $\beta$ and p75NTR (p75). The bottom right panel is the merge. The arrows indicate a triple-labeled cell. Note that virtually all of the cells express all three proteins. $\boldsymbol{F}$, Photomicrographs of SKP-derived SCs double-labeled for the genetic YFP tag, and the peripheral myelin protein $\mathrm{P}_{0}$. The top left panel is Hoechst nuclear staining to show all of the cells in the field, and the bottom right panel is the merge. The arrows indicate double-labeled cells.
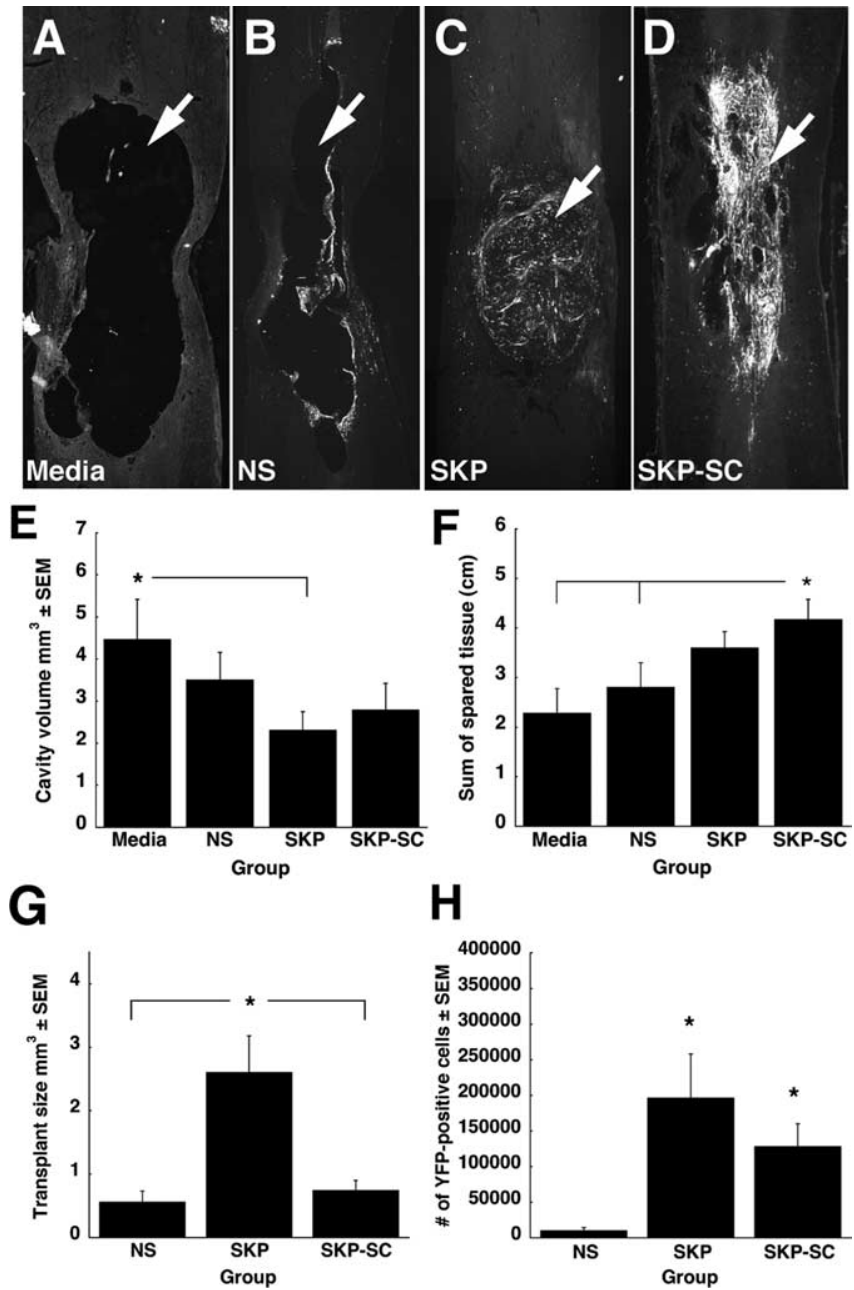

Figure 2. Cavity size and cell survival in the contused rat spinal cord after transplantation of SKPs versus SKP-derived SCS. A-D, Fluorescence, low-magnification (10X) photomicrographs of longitudinal sections through the spinal cord of rats that were transplanted 11 weeks earlier with medium alone $(\boldsymbol{A})$, YFP-expressing neonatal forebrain SVZ neurospheres $(\boldsymbol{B})$, YFPexpressing neonatal SKPS (C), or YFP-expressing SKP-derived SCS (D). $\boldsymbol{E}-\boldsymbol{G}$, Note that only SKP-derived SCS formed bridges across the longitudinal plane of the cavity with good rostrocaudal integration. Quantification of sections similar to those shown in $\boldsymbol{A}-\boldsymbol{D}$ to obtain the mean cavity size $(\boldsymbol{E})$, the amount of spared tissue $(\boldsymbol{F})$, and the transplant size $(\boldsymbol{G}) .{ }^{*} p<0.05$ relative to medium alone $(\boldsymbol{E}, \boldsymbol{F})$ and/or to neurospheres $(\boldsymbol{F}, \boldsymbol{G})$. Note that naive SKPs significantly reduced the cavity size and demonstrated the largest transplant volumes, whereas SKP-derived SCs significantly spared the tissue rim. $\boldsymbol{H}$, Number of surviving, YFP-positive cells 12 weeks after transplantation. Note that, of the three groups, SKPs had the highest survival, followed by SKP-derived SCs, both showing significantly greater survival than neurospheres. ${ }^{*} p<0.05$. In $\boldsymbol{E}-\boldsymbol{H}$, results represent mean $\pm \mathrm{SEM}$.

Analysis of longitudinal sections through the contused spinal cord revealed that SKPs, SKP-SCs, and neurospheres demonstrated distinct differences in terms of survival, integration pattern, and host tissue protection. Contusion injury typically results in the formation of a large cavity, leaving only a small rim of spared host tissue, a result that was obtained when medium alone was injected (Fig. 2A). Transplanted neurospheres were highly compromised in their survival after transplantation into the lesion cavity, and only small numbers $(<15,000)$ were found scattered along the edge of the lesion cavity (Fig. $2 B, H$ ), a finding consistent with previous studies showing that neurospheres do not survive well within the lesion site (Ogawa et al., 2002; Okano et al., 2003; Watanabe et al., 2004). However, those few neurosphere cells that entered the spared spinal cord parenchyma sur- 
vived and displayed some migration (Fig. $2 B$ ). In contrast to neurospheres, survival of SKPs and SKP-SCs was far more robust, with $\sim 180,000$ and 140,000 YFP-positive cells surviving at 11 weeks after transplantation (Fig. $2 C, D, H$ ). Transplant morphology was, however, dramatically different for SKPs versus SKPSCs. Although both reduced the degree of cavitation (Fig. 2C-E), they differed significantly in their integration into the host spinal cord. SKP-SCs consistently showed good integration into the host spinal cord, extending through the length of the spinal contusion site in a predominantly rostral-caudal alignment. Notably, there was no sharp delineation between the transplant and the host tissue rostral and caudal to the transplant, and many SKP-SCs integrated from the transplant into the spinal cord (Fig. $2 D)$. Within the transplant, the YFP-positive SKP-SCs were uniformly spindle-shaped and were densely packed (Fig. 2D). Importantly, the SKP-SCs had a significant effect on the survival and maintenance of the host tissue surrounding the lesion cavity, leading to significant sparing of the tissue rim relative to both medium alone and neurosphere transplants (Fig. $2 F$ ), a finding consistent with a neuroprotective effect.

In contrast to SKP-SCs, although transplanted SKPs filled much of the lesion cavity (Fig. 2C,E, G), there was a sharp demarcation between the transplant and the host spinal cord tissue, and the transplanted cells did not display any predominant orientation (Fig. 2C). Within the transplant, the SKPs differentiated into a variety of cell shapes, indicative of different cell types (described below), and they were packed much more loosely than the SKPSCs. Immunostaining for the proliferation marker Ki67 indicated that, at this late time point after transplantation, very few donor YFP-positive SKPs were proliferating ( $<2 \%$ ) (supplemental Fig. $1 D$, available at www.jneurosci.org as supplemental material). Thus, both SKPs and SKP-SCs survive well in the lesioned spinal cord, but only SKP-SCs provide significant sparing of host tissue surrounding the lesion.

\section{Transplanted SKP-derived SCs modify the extracellular environment after spinal cord lesion}

The observed differences in transplant morphology suggested that there were differences in integration of the transplanted cells with the host tissue, and perhaps in reactive gliosis. To address this, we performed immunocytochemical analysis for YFP (to identify transplanted cells), GFAP (to assess reactive gliosis), and rat p75NTR (to identify endogenous SCs); host astrocytes express GFAP, but not YFP or rat p75NTR, whereas cells that express rat p75NTR but not YFP will be host SCs. With regard to SKP-SC transplants, there was extensive intermingling between the transplanted cells and host astrocytes, both within the transplant and within the host tissue around the lesion (Fig. 3A). There was also extensive intermingling between transplanted SKP-SCs and host p75NTR-positive SCs (Fig. 3A) (described below). In contrast to the minimal astrocytic response to SKP-SCs, the naive SKP transplants were surrounded by a sharp border of reactive astrocytes expressing high levels of GFAP (Fig. 3C), and virtually none of the host astrocytes infiltrated into the body of the SKP transplants. This border of reactive astrocytes was interrupted by groups of invading host rat p75NTR-positive SCs (Fig. 3C), which also infiltrated into the transplant itself (described below). A similar GFAP-positive, reactive astrocyte response was seen after transplantation of neurospheres, where the reactive astrocytes lined the walls of the large cavities found in these spinal cords (Fig. 3E).

One of the consequences of the reactive astrocyte response after spinal cord injury is increased expression of chondroitinsulfate proteoglycans (CSPGs) such as neurocan. Expression of
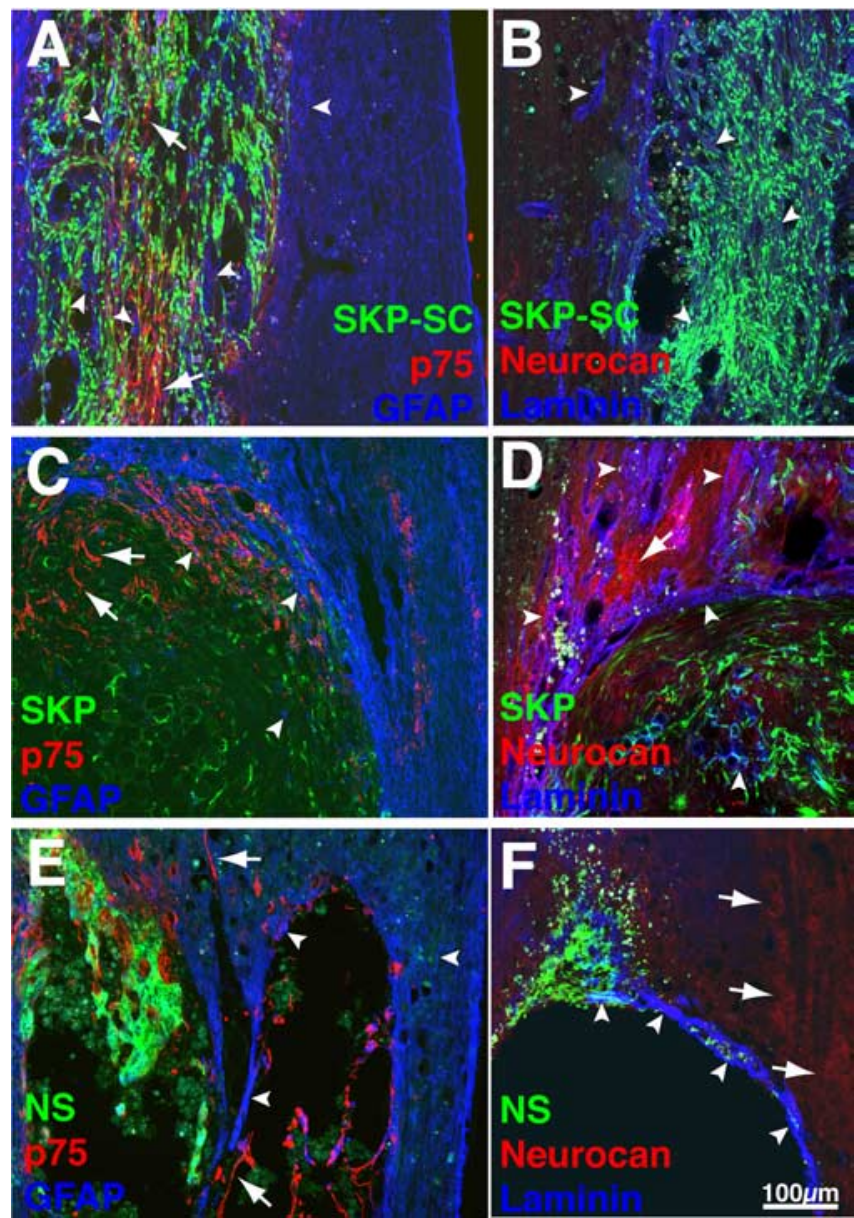

Figure 3. Transplanted SKPs and SKP-derived SCs modify the extracellular environment surrounding the lesion. $\boldsymbol{A}-\boldsymbol{F}$, Fluorescence photomicrographs of longitudinal sections through the spinal cord of rats that were transplanted 11 weeks earlier with neonatal YFP-expressing SKP-derived SCS $(\boldsymbol{A}, \boldsymbol{B})$, YFP-expressing SKPS $(\boldsymbol{C}, \boldsymbol{D})$, or YFP-expressing forebrain SVZ neurospheres (NS) $(\boldsymbol{E}, \boldsymbol{F})$, showing both the transplant and the adjacent tissue rim. $\boldsymbol{A}, \boldsymbol{C}, \boldsymbol{E}$, Immunocytochemistry for YFP expressed by the transplanted cells (green) for rat-specific 75 neurotrophin receptor (red), which is specific for host SCs, and for GFAP (blue), which, in the absence of coexpression of p75NTR, is specific for astrocytes. The arrowheads denote GFAP-positive astrocytes, and the arrows denote host SCs. Note that SKP-derived SCs (green) allow infiltration into the transplant of both astrocytes (blue) and SCs (red) from the host tissue. The same occurs, albeit to a significantly lesser extent, for transplanted SKPs. $\boldsymbol{B}, \boldsymbol{D}, \boldsymbol{F}$, Immunocytochemistry for YFP expressed by the transplanted cells (green), for neurocan (red), an inhibitory ECM molecule, and for laminin (blue). The arrows and arrowheads denote regions of neurocan and laminin expression, respectively. Note that the expression of neurocan (red) is greatly reduced in the spinal cord tissue surrounding the SKP-derived SC transplant relative to transplants of either SKPs or neurospheres. Moreover, laminin (blue) is expressed throughout the transplant and to some extent in the intact rim in spinal cords receiving SKP-derived SCs or SKPs, but is only found in the border zone lining the mostly empty cavities of neurosphere transplants.

CSPGs is considered to be one of the negative sequelae of spinal cord injury, because they inhibit axonal growth and contribute to the barrier imposed by the astrocytic scar (Fitch and Silver, 1997; Bradbury et al., 2002; Borisoff et al., 2003; Jones et al., 2003; Monnier et al., 2003; Tang et al., 2003). Given that transplantation of SKP-SCs caused only minimal reactive gliosis (Fig. $3 A$ ), we asked whether they also had an impact on expression of extracellular matrix molecules such as neurocan. To do this, we performed immunocytochemistry for YFP (to follow transplanted cells), neurocan, and laminin, the latter of which is a permissive substrate for axonal growth (Grimpe and Silver, 2002). Remarkably, levels of neurocan in the host tissue sur- 

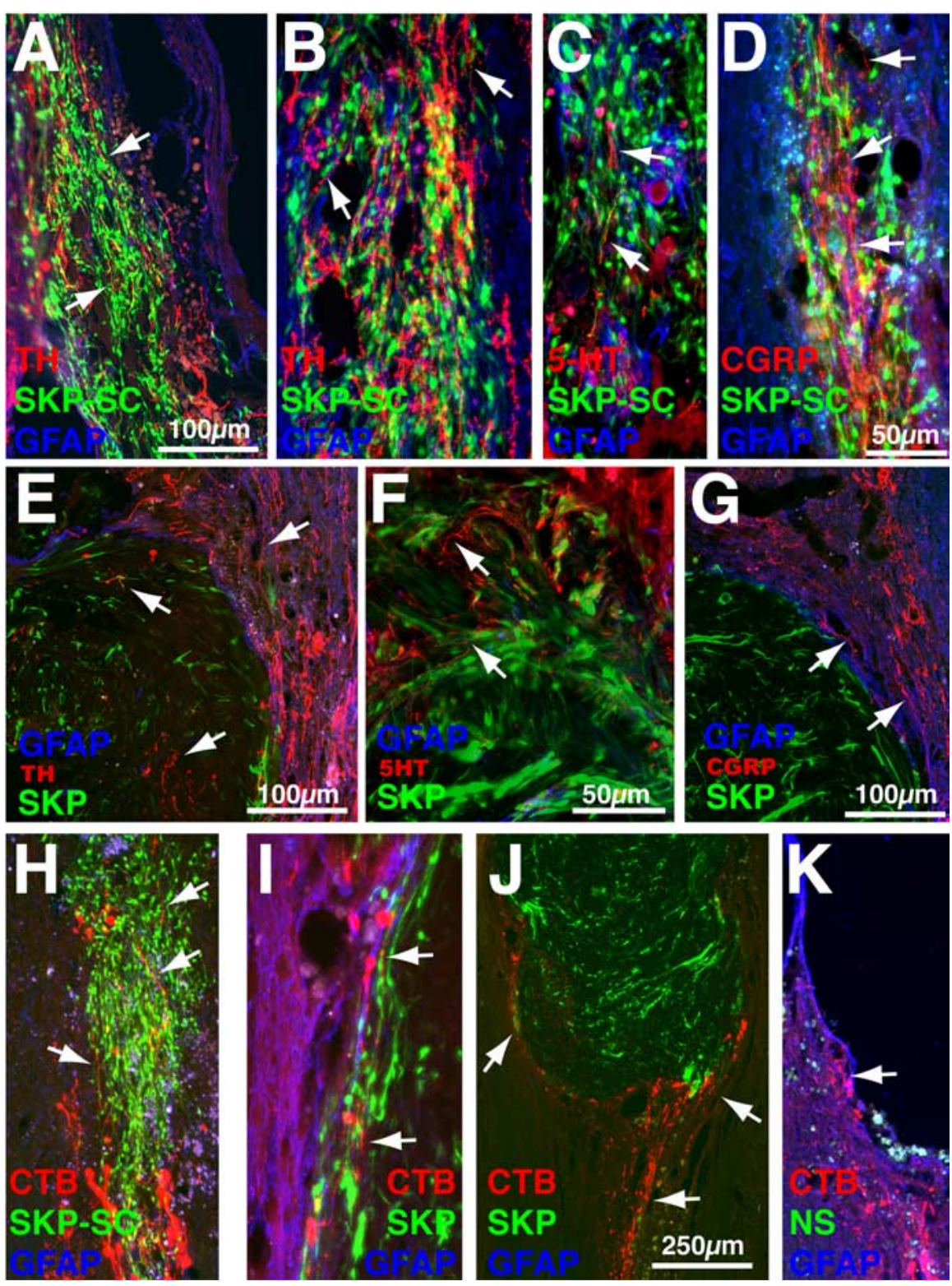

Figure 4. SKP-derived SCs promote axonal growth and sprouting into the transplant region. $\boldsymbol{A}-\boldsymbol{K}$, Fluorescence photomicrographs of longitudinal sections through the spinal cord of rats that were transplanted 11 weeks earlier with neonatal, YFPexpressing SKP-derived SCS $(\boldsymbol{A}-\boldsymbol{D}, \boldsymbol{H})$; neonatal, YFP-expressing SKPS $(\boldsymbol{E}-\boldsymbol{G}, \boldsymbol{I}, \boldsymbol{J})$; or neonatal, YFP-expressing forebrain SVZ neurospheres (NS) (K). In all cases, sections were immunostained for GFAP (blue). $\boldsymbol{A}, \boldsymbol{B}$, Immunocytochemistry for the YFP tag in the transplanted SKP-derived SCS (green) and for TH (red), a marker for descending noradrenergic fibers. $\boldsymbol{B}$ is a higher magnification image, and the arrows indicate TH-positive axons coursing through the SKP-derived SC bridge in the center of the contusion lesion. $\boldsymbol{C}, \boldsymbol{D}$, Immunocytochemistry for the YFP tag in the transplanted SKP-derived SCs (green) and either serotonin (C; 5-HT, red), a marker for descending serotonergic axons, or CGRP (D; red), a marker for sensory axons. The arrows indicate serotonin- or CGRP-positive fibers. $\boldsymbol{E}-\boldsymbol{G}$, Immunocytochemistry for the YFP tag in transplanted SKPs (green), and for tyrosine hydroxylase (E), serotonin $(\boldsymbol{F} ; 5-H T)$, or CGRP $(\boldsymbol{G})$ (red in all cases). $\boldsymbol{F}$ is a higher magnification image. The arrows denote positive axons. Note that, unlike SKP-derived SCS, the axons do not course through the transplant but instead are primarily limited to the border of the transplant. $\boldsymbol{H}-\boldsymbol{K}$, Ascending sensory axons were traced with the anterograde tracer CTB at 10 weeks after lesion, animals were killed 2 weeks later, and immunocytochemistry was performed for the YFP tag in the transplanted cells (green), and for cholera toxin B (red). Photomicrographs of longitudinal sections through the spinal cords of these animals demonstrate that ascending, CTB-positive axons grew at least $1 \mathrm{~mm}$ into transplants of SKP-derived SCS $(\boldsymbol{H})$, but not into transplants of naive SKPs $(\boldsymbol{I}, \boldsymbol{J})$ or of neurospheres $(\boldsymbol{K})$. The arrows in $\boldsymbol{H}, \boldsymbol{I}$, and $\boldsymbol{J}$ indicate the CTB-positive axons, and those in $\boldsymbol{K}$ indicate terminal bulbs of CTB-positive axons.

rounding the SKP-SC transplants, and in the transplants themselves, were very low, similar to levels seen in uninjured regions of the spinal cord (Fig. 3B). In contrast, as predicted by the reactive gliosis seen around the SKP transplants, there was a pronounced increase in neurocan expression in the host spinal cord adjacent

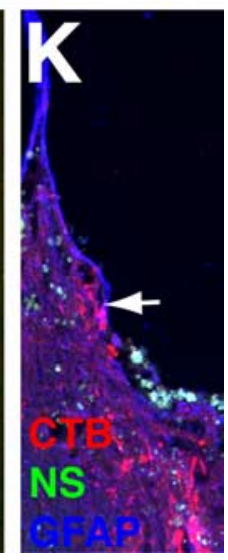

to and rostral to SKP transplants (Fig. 3D). A distinct increase in neurocan was also seen around the lesion cavities of the neurosphere-transplanted animals (Fig. $3 F)$. Differences were also observed in the laminin response between these different groups. The SKP-SC transplants displayed abundant laminin immunoreactivity within the transplant and in the surrounding host tissue. In SKP-treated spinal cords, laminin was particularly enriched in the borders of the SKP transplants, whereas only the walls of the lesion cavities contained laminin in neurosphere transplants. Thus, transplantation of SKP-SCs, but not of SKPs or neurospheres, had very positive effects on the environment of the host spinal cord, suppressing reactive gliosis and neurocan expression.

\section{SKP-derived SCs promote axonal growth and sprouting into the transplant region}

SCs, either alone or in conjunction with other therapies, have previously been shown to provide a conducive environment for the growth and regeneration of CNS axons after spinal cord injury (Paino and Bunge, 1991; Paino et al., 1994; Xu et al., 1995, 1997, 1999; Tuszynski et al., 1998; Weidner et al., 1999; Azanchi et al., 2004). We therefore characterized axonal growth in the transplant groups, analyzing both ascending and descending fibers.

Immunocytochemical analysis of the 12 week transplant group for neurofilament and tubulin revealed that SKP-SCs were regularly associated with very large numbers of axons both adjacent to and within the transplants (supplemental Fig. 2, available at www.jneurosci.org as supplemental material; or for examples, see Figs. $6 C, H, 7 A$ ). Many of these axons traversed the entire injury site in a rostrocaudal orientation, indicating either axonal preservation and/or directed growth of sprouting/regenerating axons. We then analyzed defined subsets of axons by immunocytochemistry for TH (a marker for descending noradrenergic and dopaminergic axons as well as sympathetic fibers), serotonin (5-HT, a marker for descending serotonergic axons), and CGRP (a marker for ascending sensory fibers). In addition, ascending sensory fibers were traced using CTB, which had been injected into the sciatic nerve 1 week before killing. These analyses (Fig. 4A-D,H) supported the notion that SKP-SCs promoted new axonal sprouting and/or regeneration. Specifically, we observed massive infiltration of THpositive (Fig. 4A,B) and 5-HT-positive (Fig. $4 C$ ) axonal sprouts at the rostral spinal cord/transplant interface and were able to follow these sprouts as far as $1 \mathrm{~mm}$ distally. These axons appar- 
ently originated from straight descending projections indicative of their brainstem origin and were associated with the cellular bridges formed by the transplanted SKP-SCs. Similarly, CGRPpositive sensory axons were found growing in close association with the transplanted SKP-SCs, infiltrating and extending through the lesion site in primarily a rostrocaudal orientation (Fig. $4 D$ ). Moreover, tracing of the ascending sensory axons by immunocytochemistry for CTB revealed that these axons grew for $>1 \mathrm{~mm}$ into the SKP-SC transplants (Fig. $4 H$; corresponding image of only CTB-labeled axons is provided in supplemental Fig. $3 C$, available at www.jneurosci.org as supplemental material). Thus, SKP-SCs provide an environment that is highly conducive to the maintenance and/or growth of axons.

Very different results were obtained when transplants of naive SKPs were analyzed in a similar manner. Immunocytochemical analysis for NFM and $\beta$ III-tubulin indicated the presence of many fewer axons within the SKP transplants relative to the SKP-SC transplants (supplemental Fig. $2 B$, available at www. jneurosci.org as supplemental material; for examples, see Figs. $6 E, 7 D, E)$. Immunocytochemical analysis for $\mathrm{TH}, 5-\mathrm{HT}$, and CGRP revealed that axons positive for these markers were primarily found along the host/transplant interface, with only a few extending $>500 \mu \mathrm{m}$ into the transplant (Fig. $4 E-G$ ). Of those axons that did sprout into the transplant, they were somewhat randomly oriented, presumably as a consequence of the relatively random orientation of the differentiated SKP-derived cells within the transplant region. Analysis of the ascending sensory axons by immunocytochemistry for CTB revealed that these axons failed to enter the naive SKP transplants, and instead looped around and grew along the graft-host interface (Fig. $4 J$ ). They typically ended in large terminal bulbs indicative of frustrated regeneration (Fig. 4I). Thus, SKP transplants promote limited axonal growth relative to transplanted SKP-SCs. Importantly, transplanted YFP-positive SKPs were never found to be positive for any of the neuronal markers that were analyzed (TH, 5-HT, $\beta$ III-tubulin, or NFM) indicating that naive SKPs did not differentiate into neurons within the injured spinal cord.

In contrast to both SKP-SCs and SKPs, neurospheres did not promote axonal growth, and only limited sprouting of CTBpositive axons was seen in the vicinity of the neurosphere transplants (Fig. $4 K$ ), likely because of the limited survival of these cells within the lesion site.

Although these data indicate that SKP-SCs provide a supportive axonal environment, they do not address the question of whether SKP-SCs actually enhance sprouting/regeneration of axons, as opposed to merely promoting maintenance of spared axons. To distinguish these two possibilities, we quantified the number of TH- and 5-HT-positive axons present within SKP-SC transplants at 1,2, and 11 weeks after transplant. One week after transplant, SKP-SC transplants contained $\sim 500$ and $400 \mathrm{TH}-$ and 5-HT-positive axons, respectively, numbers that were statistically similar to contused animals that were injected with medium alone (Fig. 5D,F). At 2 weeks, medium-alone animals did not exhibit a change in axon numbers from 1 week, and the axons remained primarily within astrocyte-rich regions of intact surrounding tissues (Fig. 5A). However, at 2 weeks, the TH- and 5-HT-positive axons in the SKP-SC grafts (Fig. $5 B, C$ ) showed a significant increase in number compared with 1 week after injury by approximately twofold $\left(\mathrm{TH}, t_{(10)}=2.93, p<0.02 ; 5-\mathrm{HT}, t_{(10)}\right.$ $=4.62, p<0.05)$. Remarkably, by 11 weeks after transplant, the number of TH- and 5-HT-positive axons in the SKP-SC transplants had increased another threefold to fourfold to $\sim 3000$ in both cases $\left(\mathrm{TH}, t_{(8)}=2.744, p<0.03\right.$; 5 -HT, $t_{(8)}=4.62, p<$
0.002) (Fig. $5 E, G$ ). In contrast, the neurosphere-treated animals contained only $\sim 500 \mathrm{TH}$ - and 300 5-HT-positive axons, whereas the SKP transplants contained $\sim 1000 \mathrm{TH}-$ and 3005 -HTpositive axons (Fig. $5 E, G$ ). ANOVA revealed a significant effect of treatment such that both $\mathrm{TH}\left(F_{(2,12)}=9.618 ; p<0.004\right)$ and 5 -HT $\left(F_{(2,12)}=29.08 ; p<0.0001\right)$ axons were significantly reduced in spinal cords from neurosphere- or SKP-treated compared with SKP-derived SC-treated animals $(p<0.001)$. Although not quantified, CTB-labeled ascending axons exhibited a similar increase at 2 and 11 weeks after treatment with SKP-SC relative to medium alone, neurospheres, or naive SKP (shown in supplemental Fig. 3A-C, available at www.jneurosci.org as supplemental material). This dramatic increase in the number of axons in SKP-SC transplants between 1 and 11 weeks after transplant indicates that SKP-SCs enhanced the sprouting and/or regeneration of spinal cord axons and that these axons grew in a directed rostral-caudal manner through the lesion site.

\section{Naive SKPs and SKP-derived SCs myelinate axons in the injured spinal cord}

One strategy for spinal cord repair is to promote the myelination of demyelinated, spared axons in the lesion rim, and to myelinate any axons that do sprout or regenerate. We therefore asked whether SKP-SCs or naive SKPs myelinated axons in the injured spinal cord, as they do in the regenerating peripheral nerve (McKenzie et al., 2006). To answer this question, we performed immunocytochemistry for YFP, and for $\mathrm{P}_{0}$, a myelin protein that is specific for SCs and SC-derived myelin. Alternatively, we used an antibody for MBP, another myelin protein expressed in both central and peripheral myelin. This analysis revealed the presence of many myelinating, YFP-positive SCs within transplants of both SKP-SCs and naive SKPs (Fig. 6). For both types of transplants, these YFP-positive, $\mathrm{P}_{0}$-positive, or MBP-positive cells displayed a bipolar morphology (Fig. $6 A, B$ ), consistent with a SC phenotype, and they were closely associated with and aligned along axons (Fig. 6C). In the case of SKP-SC transplants, these SCs were present throughout the lesion tissue bridge and were oriented longitudinally along the axis of the axon tracts that coursed through the transplants (Fig. 6C). SKP-SCs also migrated into the spared tissue rim around the lesion, where they myelinated spared host axons that were presumably demyelinated as a consequence of the injury (Fig. 6D). In contrast, in SKP transplants, the transplant-derived SCs were more frequently seen at the edges of the transplant, where they were randomly oriented (Fig. $6 A, B, E$ ). This localization is likely a consequence of the location and random orientation of the host axons within SKP transplants (Fig. 4); these axons are likely required to induce the naive SKPs to differentiate into SCs (McKenzie et al., 2006). Transplant-derived myelinating SCs were not observed in the host tissue around SKP transplants. With regard to neurospheres, myelination of spared host axons, presumably by transplantderived oligodendrocytes, was observed only very occasionally (Fig. 6F).

To demonstrate that the transplant-derived $\mathrm{P}_{0}$-positive, MBP-positive SCs were making bona fide compact myelin, we performed immunocytochemistry for the axonal proteins Kv1.2 and Caspr, both of which localize to the Nodes of Ranvier on myelinated axons (Rasband et al., 1998; Rasband and Trimmer, 2001a,b). This analysis revealed that axons associated with YFPpositive myelinating SCs demonstrated appropriate clustering of these two paranodal proteins in both SKP and SKP-SC transplants (Fig. 6G,H).

To quantify the number of myelinating cells generated by 

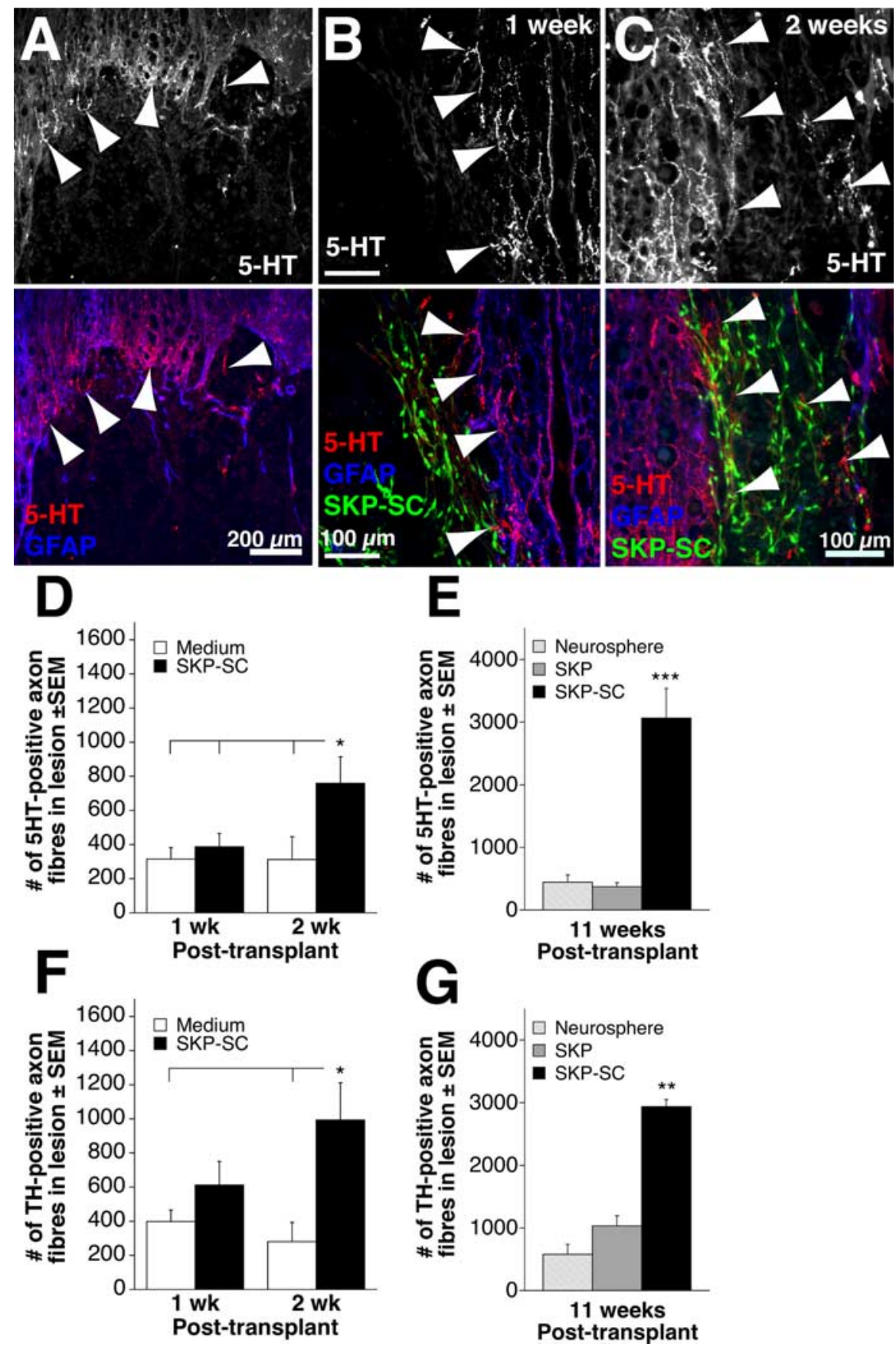

Figure 5. SKP-SCs promote robust axonal growth in the injured spinal cord. $\boldsymbol{A}$, Top panel shows 5-HT-immunolabeled axons 2 weeks after medium-only injection into the contusion site. 5-HT-positive axon fibers (red; arrowheads) are mostly limited to the areas of intact CNS tissue rich with GFAP-positive astrocytes (blue; bottom panel). B, One week after SKP-derived SCs transplant, 5-HT-positive axons (top panel) are primarily limited to intact host tissue (GFAP, blue; bottom panel). C, In contrast, after 2 weeks, many more 5-HT-positive axons (top panel; arrowheads) are observed within SKP-derived SC transplants, likely an indication of axonal preservation or enhanced sprouting. Note that 5-HT fibers are observed even in areas that do not contain GFAP-positive astrocytes (blue; bottom panel). D, Quantification of 5-HT-positive axons within transplants of SKP-SCS at 1 and 2 weeks after injury. No differences in axon number were observed after 1 week; however, by 2 weeks after transplant, SKP-derived SCS supported a twofold increase in axon number relative to medium-alone controls. ${ }^{*} p<0.05$. $\boldsymbol{E}$, Quantification of 5-HT-positive axons in transplants of neurospheres, SKPs, or SKP-derived SCs (as shown in Fig. 4) 11 weeks after transplantation. Treatment with SKP-derived SCs resulted in a fourfold increase in 5-HT axon numbers relative to both SKPs and neurospheres. ${ }^{* * *} p<0.001 . \boldsymbol{F}$, Quantification of TH-positive axons within the transplants of SKP-derived SC s at 1 and 2 weeks after injury. There was no difference in TH-positive axon numbers after 1 week; however, by 2 weeks after transplant, SKP-derived SCs supported a threefold increase compared with medium-alone controls and a significant increase compared with SKP-derived SC transplants at 1 week. ${ }^{*} p<$ 0.05. G, Quantification of TH-positive axons in transplants of neurospheres, SKPs, or SKP-derived SCs (as shown in Fig. 4) 11 weeks after transplantation. After 11 weeks, SKP-derived SC transplant supported a threefold increase in axon number relative to SKP-or neurosphere-treated animals. ${ }^{* *} p<0.01$. All group analyses were $n=5$ with the exception of 1 week medium controls $(n=3)$. All data are group means \pm SEM. transplants of SKPs versus SKP-SCs, we counted the number of YFP-positive cells that generated a $\mathrm{P}_{0}$-positive myelin sheath (for examples of what was counted, see Fig. $6 C$, right; $D$, right). These counts demonstrated that spinal cords transplanted with SKP-SCs and SKPs contained 30,00035,000 and 15,000 transplant-derived myelinating SCs, respectively (Fig. 6J). This represented $\sim 35$ and $15 \%$ of the total YFP-positive cells within these transplants (Fig. 6I). Thus, a large percentage of SKPSCs myelinated both spared and sprouting or potentially regenerating host axons. Interestingly, host axons also apparently provided the cues necessary to induce a subset of naive SKPs to differentiate into myelinating SCs, a phenomenon we observed previously (McKenzie et al., 2006).

This quantification also indicated that the large majority of naive SKPs did not differentiate into a myelinating SC phenotype, and our analysis with NFM/tubulin indicated that they also do not differentiate into neurons. We therefore asked whether they differentiated into mesodermal phenotypes within the environment of the injured spinal cord, performing immunocytochemistry for smooth muscle actin and collagen type II, both mesodermal markers. We also examined the transplants morphologically for adipocytes, a cell type SKPs are known to generate (Toma et al., 2001). This analysis revealed that naive SKPs generated both smooth muscle actin- and collagen type II-positive cells, as well as cells with the morphology of adipocytes (supplemental Fig. $1 A-C$, available at www.jneurosci.org as supplemental material). In contrast, transplanted SKP-SCs were never observed to express any inappropriate markers, and all of the cells maintained an appropriate SC morphology with many expressing p75 (supplemental Fig. 4, available at www. jneurosci.org as supplemental material) and/or $\mathrm{P}_{0}$, but not detectable levels of GFAP or $\mathrm{S} 100 \beta$.

\section{SKPs and SKP-derived SCs promote} recruitment of endogenous, myelinating SCs into the injured spinal cord

During our analysis of transplant-derived myelinating SCs, it became evident that SKP and SKP-SC transplants were promoting robust recruitment of endogenous SCs, because we observed many YFPnegative, $\mathrm{P}_{0}$-positive cells. We therefore analyzed this recruitment of endogenous SCs, which has been previously described to be enhanced by transplants after spinal cord injury (Ramer et al., 2004a; Hill et al., 2006). Analysis of SKP-SC transplants re- 
vealed that both the body of the transplant and the spared tissue rim contained many YFP-negative, $\mathrm{P}_{0}$-positive myelin sheaths associated with endogenous host axons (Fig. 7A). A similar pattern of immunostaining was observed when sections were immunostained for rat-specific p75NTR, which recognizes host, but not transplanted SCs (Fig. 7 B, C). YFP-negative, $\mathrm{P}_{0^{-}}$ positive myelin sheaths were also encountered in both the SKP transplants and in the neurosphere transplants. In the case of SKP transplants, these endogenous SCs were primarily limited to the exterior part of the transplants and were not as obvious in the tissue rim (Fig. 7D-F). Similar results were obtained in the case of the neurospheres and after medium control injections, although the density of host SCs appeared much lower (neurospheres shown in Fig. 6G-I).

To more quantitatively assess this recruitment, we counted YFP-negative cells that displayed a $\mathrm{P}_{0}$-positive myelin sheath. This analysis revealed that, of the myelinating SCs in the spinal cords of SKP and SKP-SC transplanted animals, $\sim 80$ and $53 \%$ of the cells were YFP-negative, respectively, and thus were presumably recruited from the host tissue (Fig. 7J). When these numbers were analyzed with regard to endogenous SCs in the contusion lesion versus the spared rim, it became apparent that equal numbers of endogenous myelinating SCs were present in the lesion cavities transplanted with SKPs versus SKP-SCs $(\sim 60,000$ per spinal cord in both cases) (Fig. $7 L$ ) but that SKP-SC transplants recruited significantly more endogenous myelinating SCs into the spared tissue $\operatorname{rim}(\sim 30,000$ cells per spinal cord $)$ (Fig. $7 L$ ). A similar analysis of the neurosphere transplants and the medium-alone group revealed that endogenous SCs were recruited in both cases, but that the numbers were significantly lower than for transplants of SKPs and SKP-SCs (Fig. $7 K, L)$. Thus, not only do transplanted SKP-SCs and SKPs directly generate myelinating SCs but they also recruit endogenous myelinating SCs both into the lesion cavity and into the spared tissue rim.

\section{SKP-derived SCs promote functional improvement after contusion injury}

These results indicated that SKP-derived SCs promoted tissue sparing, remyelination, and axonal growth after contusion injury. We therefore asked whether they also promoted functional locomotor recovery. To address this question, locomotor function was tested before the injury, and then at regular intervals up to 9 weeks after injury. After contusion injury, all transplanted animals displayed gross motor impairment showing only joint movement for the first couple of days and no weight-supported

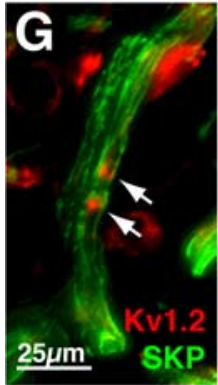

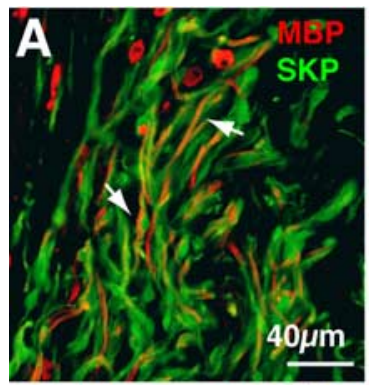
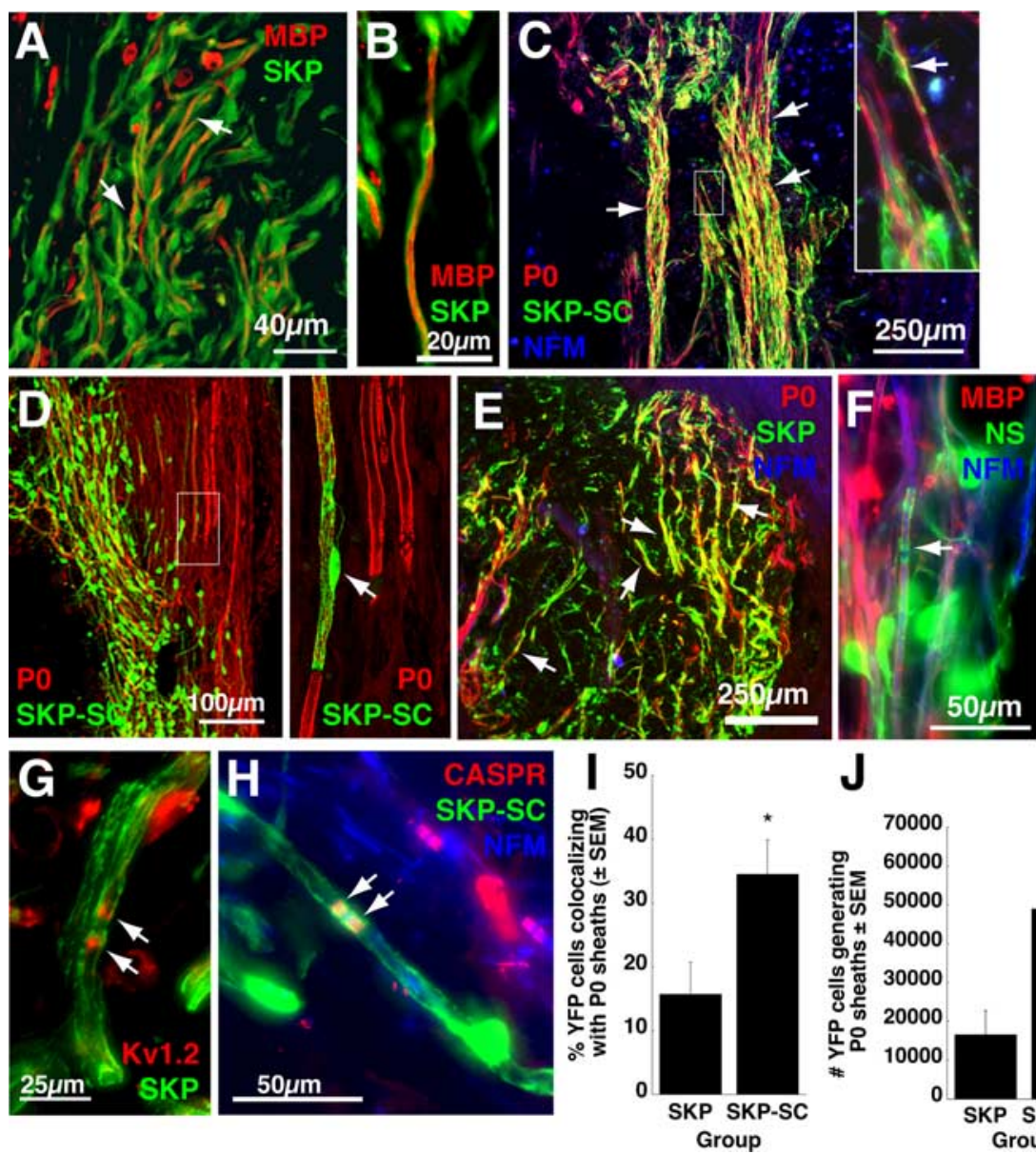

Figure 6. Naive SKPs and SKP-derived SCS myelinate axons in the injured spinal cord. Analysis of longitudinal sections of the spinal cord of animals transplanted with neonatal, YFP-expressing SKPs (SKP), SKP-derived SCS (SC), or neurospheres (NS) 11 weeks earlier. $\boldsymbol{A}, \boldsymbol{B}$, Double-label immunocytochemistry for the YFP tag in transplanted SKPs (green) and for the myelin protein of the YFP-positive, MBP-positive cells. C, Triple-label immunocytochemistry for the YFP tag in transplanted SKP-SCs (green), for $P_{0}(r e d)$, and for the axonal marker NFM (blue; the arrows indicate double-labeled $P_{0}$-positive SKP-SCS). The inset shows a high nous axon within the transplant. $\boldsymbol{D}$, Low (left panel) and high (right panel) magnification confocal micrographs of a section double-labeled for the YFP tag in the transplanted SKP-SCS (green), and for the SC-specific myelin protein $\mathrm{P}_{0}$ (red). Note that, in these patches of SC myelin derive from YFP-positive transplanted SKP-SCS, as shown in the right panel (arrows). $\boldsymbol{E}$, Triple-label as indicated by triple labeling for the YFP tag (green), the myelin-specific protein MBP (red), and the axonal marker NFM (blue). $\boldsymbol{G}$ d; $\boldsymbol{H}$; arrows). Quantification of the number $(\boldsymbol{I})$ and percentage $(\boldsymbol{J})$ of surviving, YFP-positive transplanted cells that coexpressed the peripheral myelin-specific protein $\mathrm{P}_{0}$ and associated with endogenous axons. Note that $\sim 35$ and $15 \%$ of SKP-SCS and SKPs, respectively, made $P_{0}$-positive myelin sheaths. ${ }^{*} p<0.05$. Data represent mean $\pm S E M$.

stepping for the first week (BBB $<10$ ), and partial improvement over the ensuing 9 weeks after injury (Fig. $8 A$ ). After 7 weeks after injury, most animals had regained hindlimb stepping without forelimb/hindlimb coordination. However, unlike naive SKP- or neurosphere-transplanted animals, which appeared to plateau at 7 weeks, the animals transplanted with SKP-SCs showed additional improvement, many exhibiting coordinated forelimb/ hindlimb stepping, a critical signpost of recovery from spinal cord injury. Indeed, 6 of 13 animals treated with SKP-SCs exhibited consistent stepping with at least occasional forelimb/hindlimb coordination (BBB score $\geq 12$ ), whereas only 2 of 11 and 4 of 12 animals reached this level of functionality after treatment 

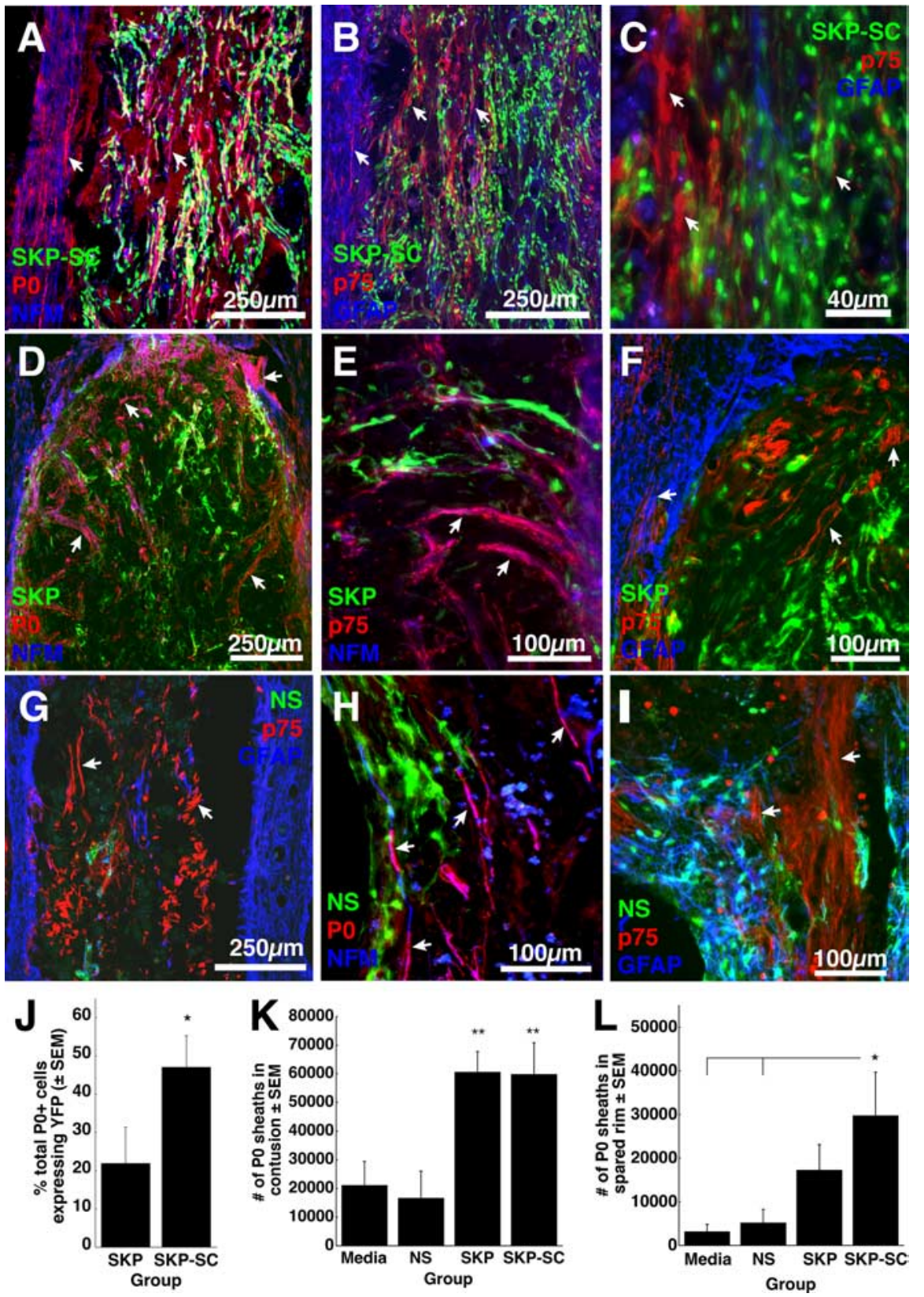

Figure 7. SKPs and SKP-derived SCs promote recruitment of endogenous SCs into the injured spinal cord. Analysis of longitudinal sections of the spinal cord of animals transplanted with neonatal YFP-tagged SKPs, SKP-derived SCS (SC), or forebrain SVZ neurospheres (NS) 11 weeks earlier. $\boldsymbol{A}$-C, Fluorescence photomicrographs of spinal cord sections immunolabeled for the YFP tag in transplanted SKP-SCs (green), for GFAP (blue), and for either $\mathrm{P}_{0}(\boldsymbol{A})$ or rat $\mathrm{p} 75 \mathrm{NTR}(\boldsymbol{B}, \boldsymbol{C})$ (both red). $\boldsymbol{C}$ is a higher magnification image. $\boldsymbol{D}-\boldsymbol{F}$, Immunolabeling of SKP transplants for the YFP tag (green), the axonal protein NFM (NF; blue), and either $P_{0}(\boldsymbol{D})$ or p75NTR $(\boldsymbol{F}) . \mathbf{G}-\boldsymbol{I}$, Immunolabeling of neurosphere transplants for the YFP tag (green), GFAP $(\boldsymbol{G}, \boldsymbol{I})$ or NFM (H) (both blue), and rat $\operatorname{p75NTR}(\boldsymbol{G}, \boldsymbol{I})$ or $\mathrm{P}_{0}(\boldsymbol{H})$ (both red). In all panels, the arrows denote host SCs that are YFP-negative and $\mathrm{P}_{0}$-positive or YFP-negative and rat p75NTR-positive. J, Quantification of the percentage of $P_{0}$-positive myelin sheaths double-labeled for the YFP tag present in transplanted SKPs or SKP-derived SCs. The other $\mathrm{P}_{0}$-positive myelin sheaths derive from endogenous SCs that have migrated into the injured spinal cord. $\boldsymbol{K}, \boldsymbol{L}$, Quantification of the total number of $\mathrm{P}_{0}$-positive myelin sheaths within the lesion site $(\boldsymbol{K})$ and within the spared rim tissue $(\boldsymbol{L}) 11$ weeks after transplantation with neurospheres, SKPs, SKP-derived SCs, or medium alone. Note that both SKPs and SKP-derived SCS recruit substantially more endogenous SCs than do transplants of neurospheres or medium alone. ${ }^{*} p<0.05 ;{ }^{* *} p<0.05$ relative to both medium control and neurosphere-treated animals. In $\boldsymbol{J}-\boldsymbol{L}$, results represent mean \pm SEM.

with neurospheres or SKP, respectively. At week 9 after injury, post hoc tests revealed a modest, but significant improvement in gross motor performance in animals receiving SKP-SCs relative to naive SKPs $\left(t_{(27)}=1.852 ; p<0.04\right)$ (Fig. $\left.8 A\right)$, but there was no statistical difference between SKP-derived SCs and neurosphere-treated animals.

To further examine specific locomotor components (i.e., paw position, toe clearance, trunk control, and tail position), we used the Basso subscoring scale (Basso, 2004). Repeated-measures ANOVA revealed a significant interaction of treatment and time $\left(F_{(6,32)}=8.10 ; p<0.001\right)$. In parallel with the gross motor measure, animals treated with SKP-SCs began to show persistent functional gains between 7 and 9 weeks after injury, which was not observed in either SKP- or neurospheretransplanted animals (Fig. $8 \mathrm{~B}$ ). Within the SKP-SC group, 8 of 14 animals achieved locomotor subscores of $\geq 5$, whereas only 3 of 11 neurosphere-transplanted and 2 of 14 SKP-transplanted animals achieved this level of motor function. At 9 weeks after injury, $\operatorname{ANOVA}\left(F_{(2,37)}=5.14 ; p<0.02\right)$ and group comparisons confirmed that SKP-SC motor performance was significantly improved relative to both SKPtreated $(p<0.005)$ and neurospheretreated $(p<0.05)$ groups. In addition, we assessed hindlimb placing and stepping errors in the horizontal-ladder test (Metz and Whishaw, 2002). Although the SKP-SC group made fewer errors per step than the SKP and neurosphere groups at 5 and 9 weeks after injury, this difference did not reach significance (Fig. 8C).

Neuropathic pain after transplantation for spinal cord injury is of particular importance in light of a recent report suggesting that undifferentiated neural stem cells, but not predifferentiated progeny, resulted in an increased incidence of allodynia (Hofstetter et al., 2005). To ask whether SKP and SKP-SC transplants had any effect on the development of neuropathic pain, we first used the dynamic plantar aesthesiometer test to assess hindlimb withdrawal reflexes in response to mechanical stimulation (Fig. 9A). Interestingly, Kruskal-Wallis test revealed a significant effect of treatment at 5 weeks $\left[\chi^{2}(2)=9.35 ; p<0.01\right]$ and 7 weeks $\left[\chi^{2}(2)=6.237 ; p<0.05\right]$ after injury. At 5 weeks after injury, both neurosphere $(26.2 \pm 4.4 \mathrm{~g})$ and SKP-transplanted animals $(18.8 \pm 2.8 \mathrm{~g})$ showed a significant reduction in withdrawal thresholds compared with either their individual preinjury thresholds $(p<0.05)$ or with the SKP-SC transplanted group $(35.1 \pm 3.2 \mathrm{~g})$ $(p<0.05)$. Although this hypersensitivity had resolved by 9 weeks, this transient increase was potentially indicative of increased sensitivity to mechanical stimuli in the two groups transplanted with undifferentiated precursor cells. To pursue this further, we used the infrared heat test to assess sensitivity to temperature. Repeated-measures 
ANOVA revealed significant effect of treatment $\left(F_{(2,37)}=8.06 ; p<0.001\right)$ and an interaction of treatment by time $\left(F_{(2,70)}=2.33 ; p<0.04\right)$. Interestingly, neither SKP-SC- or neurospheretransplanted animals exhibited a change in withdrawal thresholds to heat at any point (Fig. $8 \mathrm{~B}$ ). However, animals treated with naive SKPs showed significantly heightened sensitivity at both 7 weeks $(p<0.05)$ and 9 weeks $(p<0.05)$ relative to either their preinjury baseline, or to both SKPSC- or neurosphere-treated animals ( $p<$ $0.05)$. Thus, two independent measures demonstrated that transplanted SKP-SCs had no impact on sensitivity to sensory stimuli but that naive SKPs produced heightened sensory responses (i.e., lowered threshold), potentially indicating the development of neuropathic pain.

\section{Discussion}

Here, we have performed a direct comparison of the neuroanatomical and functional outcomes after transplantation of SKPs, purified SKP-derived SCs, and CNS SVZ neurospheres into the contused rat spinal cord. This comparison provides strong evidence that SKP-SCs are a highly suitable transplant candidate for treatment of spinal cord injury. In particular, we demonstrate that transplanted SKPs and SKP-SCs display enhanced survival related to CNS neural stem cells, and fill or bridge the lesion cavity in the contused spinal cord. Transplanted naive SKPs also responded to endogenous cues in the injured spinal cord and differentiate into SCs that myelinate spared and/or regenerating axons. However, despite this myelination, naive SKPs do not appear to be good candidates for spinal cord repair, because they also differentiate into mesodermal cell types, cause long-term enhanced temperature sensitivity, and do not significantly enhance functional locomotor recovery relative to CNS stem cells. In contrast, transplantation of isolated, expanded SKP-SCs resulted in a more than threefold increase in myelination relative to naive SKPs. These SKP-SC transplants maintained a rostral-caudal orientation, thereby providing an effective "bridge" across the lesion cavity, which supported robust axonal growth. SKP-SC transplants also promoted sparing of the tissue rim surrounding the lesion, and caused only minimal reactive gliosis. These positive neuroanatomical effects were associated with a significant improvement in locomotor function, with no alterations in mechanical or heat sensitivity. Thus, SKPs, predifferentiated into their SC progeny, represent a potentially autologous source of precursors for treatment of spinal cord injury.

Spinal cord contusion injury triggers a cascade of secondary events that lead to neuronal and oligodendroglial death and loss of tissue adjacent to the lesion over a period of hours to weeks (Crowe et al., 1997; Hausmann, 2003; Jones et al., 2005; Schwab et al., 2006). In the wake of this process, a central cavity forms surrounded by an astrocytic scar, often leaving a small rim of spared white matter with focal demyelination (Bunge et al., 1993). Therapies aimed to treat this type of injury have focused on four different strategies: neuroprotection to reduce the size of the lesion, promotion of long-distance axon regeneration and reconnection, neural replacement/gray matter reconstitution, and remyelination of the spared axons (Enzmann et al., 2006). In this regard, reconstituting conduction across demyelinated axons is currently considered to be of particularly high priority (Blight, 2002), because substantial functional restoration can be achieved using this approach (Felts and Smith, 1992; Waxman et al., 1994; Guest et al., 1997). Here, we focused on remyelination using SKPs, an accessible dermal source of self-renewing neural crestlike precursors (Toma et al., 2001, 2005; Fernandes et al., 2004) that can generate myelinating SCs (McKenzie et al., 2006). Although SCs are a PNS glial cell, they myelinate CNS axons (Blakemore and Franklin, 1991; Gilmore and Sims, 1993), provide a highly conducive environment for axon regeneration, and enhance functional recovery when transplanted into the injured spinal cord (Guest et al., 1997; Xu et al., 1997; Keirstead et al., 1999; Xu et al., 1999; Pinzon et al., 2001; Pearse et al., 2004a,b). Our findings indicate that SKP-SCs myelinate both spared and sprouting/regenerating axons in the injured spinal cord, and promote functional locomotor recovery.

Data reported here indicate that both SKPs and SKP-SCs remyelinated the spinal cord but that significant functional improvement was only seen with SKP-SCs. This behavioral difference is likely attributable to a combination of reparative or protective effects observed only after treatment with SKP-SCs. First, the magnitude and location of remyelination differed between these two groups; SKP-SCs provided increased myelination relative to SKPs, and this myelination occurred both within the transplant and in the spared tissue rim, whereas myelination by naive SKPs was restricted to the transplant. Second, SKP-SCs provided significant preservation of tissue surrounding the lesion epicenter. Third, SKP-SCs modified the surrounding host environment, reducing reactive gliosis and decreasing neurocan expression. This was accompanied by migration of SKP-SCs into the surrounding host tissue, where they intermingled with host astrocytes at the lesion/host interface, apparently allowing a reciprocal movement of astrocytes into the transplant. Interestingly, this differs from transplants of mature nerve-derived SCs, where donor SCs and highly hypertrophied host astrocytes maintain discrete domains (Pearse et al., 2004b). This may occur because the SKP-SC population includes newly developed SCs and perhaps even SC precursors. Recent work indicates that immature versus mature SCs have very distinctive properties (Jessen and Mirsky, 2002, 2005; Wanner et al., 2006) and these features 

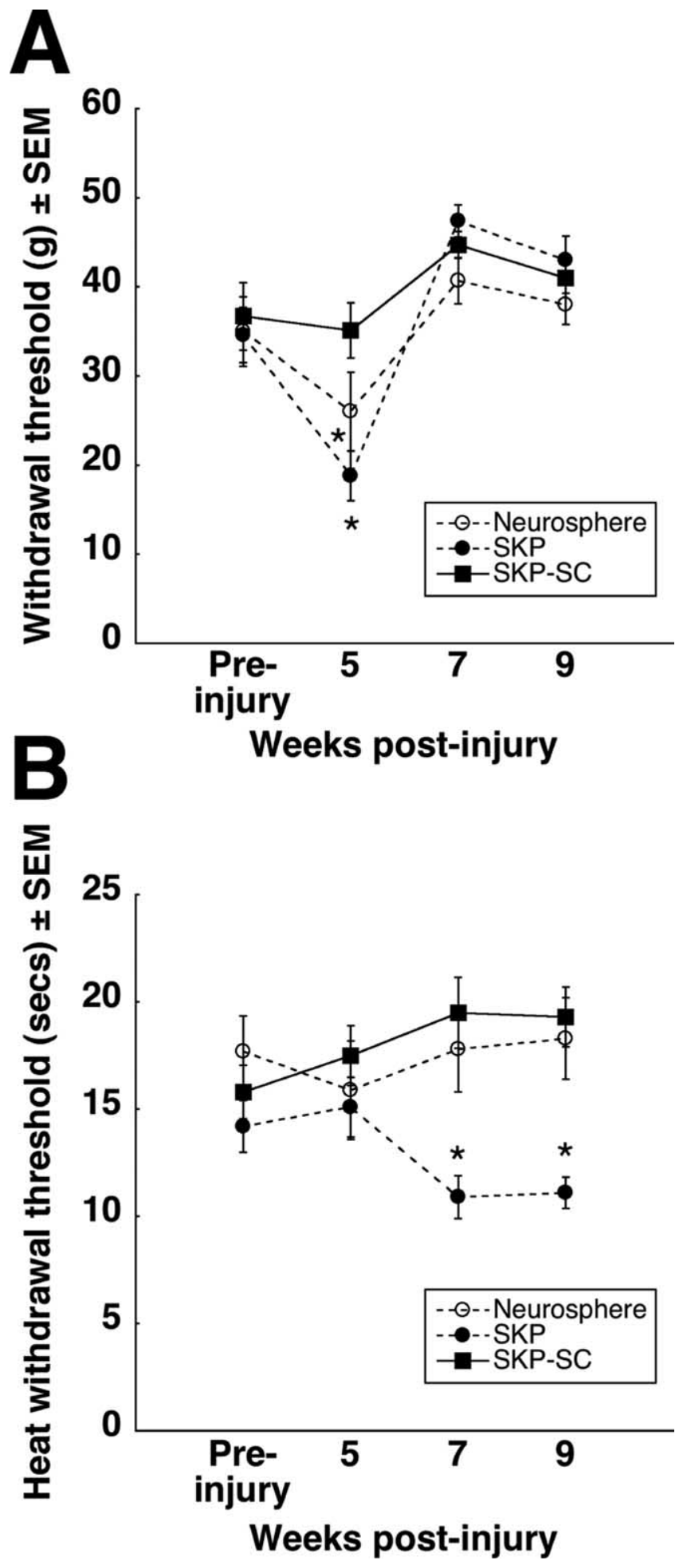

Figure 9. SKP-derived SCS do not reduce sensory thresholds when transplanted into the contused spinal cord. Animals were assessed for hindlimb sensory thresholds before their injury, and 8 weeks after transplantation of neonatal SKPs, SKP-derived SCs, or forebrain SVZ neurospheres. $\boldsymbol{A}$, Results of the mechanical sensitivity test demonstrating that transplantation of SKP-derived SCs had no impact on mechanical pain thresholds. Interestingly, transplants of SKPs or neurospheres led to a transient reduction in mechanical thresholds that recovered to preinjury levels by 9 weeks after injury. $\boldsymbol{B}$, Results of the thermal sensitivity. Analysis of the data by repeated-measures ANOVA revealed a significant week by treatment interaction $(p<0.03)$ and a main effect for treatment group $(p<0.01)$. Transplantation of SKP-derived SCS or neurospheres had no impact on sensitivity to heat, but transplantation of SKPs led to a significantly heightened sensitivity to heat at 7 weeks after injury that persisted at 9 weeks. In both panels, results represent mean \pm SEM. ${ }^{*} p<0.05$. may contribute to the enhanced axonal growth and reduced SC/ astrocyte segregation observed here. Pacifying effects on the host astrocytes have also been observed after transplantation of olfactory ensheathing cells (Ramer et al., 2004a,b).

Previous studies have used other stem cells in an attempt to remyelinate the injured spinal cord, and several of these have shown concomitant recovery of locomotor function, including CNS neural stem cells (Ogawa et al., 2002; Vroemen et al., 2003; Cummings et al., 2005; Hofstetter et al., 2005; KarimiAbdolrezaee et al., 2006), glial-restricted precursors (Cao et al., 2005), and embryonic stem (ES) cells differentiated to either a neural (Liu et al., 2000) or oligodendroglial fate (Keirstead et al., 2005; Nistor et al., 2005). These different sources all have distinct advantages and disadvantages. CNS neural stem cells, when genetically transduced with neurogenin-2 (Hofstetter et al., 2005), administered coincidentally with growth factors (KarimiAbdolrezaee et al., 2006), or transfected to express growth factors (Cao et al., 2005; Macias et al., 2006), demonstrated remyelination of the injured cord and enhanced functional recovery. However, naive CNS neural stem cells, like the naive SKP transplants performed here, caused allodynia (Hofstetter et al., 2005). ES cells, including human ES cells, can efficiently generate oligodendrocytes and their precursors appear to remyelinate the injured cord and promote functional recovery (Keirstead et al., 2005), but one major issue associated with their use is that differentiated but unpurified ES cell transplants can cause tumor formation within the environment of the CNS (Roy et al., 2006).

The studies here indicate that SKP-SCs are a viable alternative that have a number of advantages. First, SKPs derive from the dermis and thus represent an accessible, potentially autologous tissue source. In contrast, both ES cells and CNS neural stem cells are currently heterologous cell sources, and nerve-derived SCs must be harvested by invasive nerve biopsies. Second, SKPs represent an adult human precursor population, the use of which circumvents potential ethical issues. Third, human SKPs can be robustly expanded (Joannides et al., 2004; Toma et al., 2005), and SKP-SCs behave like immature, developing SCs, in contrast to nerve-derived SCs, which are mature and thus have limited proliferation potential. However, despite these potential advantages, although human SKPs can be isolated from both neonatal (Toma et al., 2005) and adult (Joannides et al., 2004; Toma et al., 2005) skin, and although human SKPs make SCs (Toma et al., 2005; McKenzie et al., 2006), we have yet to develop protocols for successful isolation and expansion of SCs from human skin.

The studies reported here reinforce the growing consensus that naive stem cells are not the best alternative for nervous system therapies. Although transplanted naive SKPs differentiated into SCs, they also differentiated into mesodermal cell types, as they do in culture. SC differentiation likely occurred in response to cues deriving from demyelinated or newly growing axons, something that we documented previously (McKenzie et al., 2006). The mesodermal differentiation likely also occurred in response to the many growth factors/cytokines that are present within the lesioned spinal cord (Nakamura and Bregman, 2001; Widenfalk et al., 2001; De Biase et al., 2005). A second undesirable outcome seen with transplanted naive SKPs was enhanced sensitivity to sensory stimuli, as was previously observed with CNS neural stem cells (Hofstetter et al., 2005). Thus, our findings, in concert with studies on neural stem cells (Hofstetter et al., 2005; Macias et al., 2006) and ES-derived cells (Roy et al., 2006), indicate that the best strategy for nervous system transplantation likely involves stem cell predifferentiation and subsequent purification of the desired cell type. 
In summary, our results indicate that transplantation of SKPSCs represent a viable alternative strategy for repairing the injured spinal cord, thus identifying a novel, accessible, and potentially autologous source of myelinating cells for nervous system repair.

\section{References}

Akiyama Y, Radtke C, Kocsis JD (2002) Remyelination of the rat spinal cord by transplantation of identified bone marrow stromal cells. J Neurosci 22:6623-6630.

Azanchi R, Bernal G, Gupta R, Keirstead HS (2004) Combined demyelination plus Schwann cell transplantation therapy increases spread of cells and axonal regeneration following contusion injury. J Neurotrauma 21:775-788.

Basso DM (2004) Behavioral testing after spinal cord injury: congruities, complexities, and controversies. J Neurotrauma 21:395-404.

Basso DM, Beattie MS, Bresnahan JC (1995) A sensitive and reliable locomotor rating scale for open field testing in rats. J Neurotrauma 12:1-21.

Biernaskie J, McKenzie IA, Toma JG, Miller FD (2006) Isolation of skinderived precursors (SKPs) and differentiation and enrichment of their Schwann cell progeny. Nat Protoc 1:2803-2812.

Blakemore WF, Franklin RJ (1991) Transplantation of glial cells into the CNS. Trends Neurosci 14:323-327.

Blight AR (2002) Miracles and molecules-progress in spinal cord repair. Nat Neurosci 5 [Suppl]:1051-1054.

Borisoff JF, Chan CC, Hiebert GW, Oschipok L, Robertson GS, Zamboni R, Steeves JD, Tetzlaff W (2003) Suppression of Rho-kinase activity promotes axonal growth on inhibitory CNS substrates. Mol Cell Neurosci 22:405-416.

Bradbury EJ, Moon LD, Popat RJ, King VR, Bennett GS, Patel PN, Fawcett JW, McMahon SB (2002) Chondroitinase ABC promotes functional recovery after spinal cord injury. Nature 416:636-640.

Bunge MB, Pearse DD (2003) Transplantation strategies to promote repair of the injured spinal cord. J Rehabil Res Dev 40:55-62.

Bunge RP, Puckett WR, Becerra JL, Marcillo A, Quencer RM (1993) Observations on the pathology of human spinal cord injury. A review and classification of 22 new cases with details from a case of chronic cord compression with extensive focal demyelination. Adv Neurol 59:75-89.

Cao Q, Xu XM, Devries WH, Enzmann GU, Ping P, Tsoulfas P, Wood PM, Bunge MB, Whittemore SR (2005) Functional recovery in traumatic spinal cord injury after transplantation of multineurotrophin-expressing glial-restricted precursor cells. J Neurosci 25:6947-6957.

Craig CG, Tropepe V, Morshead CM, Reynolds BA, Weiss S, van der Kooy D (1996) In vivo growth factor expansion of endogenous subependymal neural precursor cell populations in the adult mouse brain. J Neurosci 16:2649-2658.

Crowe MJ, Bresnahan JC, Shuman SL, Masters JN, Beattie MS (1997) Apoptosis and delayed degeneration after spinal cord injury in rats and monkeys. Nat Med 3:73-76.

Cummings BJ, Uchida N, Tamaki SJ, Salazar DL, Hooshmand M, Summers R, Gage FH, Anderson AJ (2005) Human neural stem cells differentiate and promote locomotor recovery in spinal cord-injured mice. Proc Natl Acad Sci USA 102:14069-14074.

David S, Aguayo AJ (1981) Axonal elongation into peripheral nervous system "bridges" after central nervous system injury in adult rats. Science 214:931-933.

De Biase A, Knoblach SM, Di Giovanni S, Fan C, Molon A, Hoffman EP, Faden AI (2005) Gene expression profiling of experimental traumatic spinal cord injury as a function of distance from impact site and injury severity. Physiol Genomics 22:368-381.

Deumens R, Koopmans GC, Joosten EA (2005) Regeneration of descending axon tracts after spinal cord injury. Prog Neurobiol 77:57-89.

Dietz V, Curt A (2006) Neurological aspects of spinal-cord repair: promises and challenges. Lancet Neurol 5:688-694.

Enzmann GU, Benton RL, Talbott JF, Cao Q, Whittemore SR (2006) Functional considerations of stem cell transplantation therapy for spinal cord repair. J Neurotrauma 23:479-495.

Felts PA, Smith KJ (1992) Conduction properties of central nerve fibers remyelinated by Schwann cells. Brain Res 574:178-192.

Fernandes KJ, Kobayashi NR, Gallagher CJ, Barnabe-Heider F, Aumont A, Kaplan DR, Miller FD (2006) Analysis of the neurogenic potential of multipotent skin-derived precursors. Exp Neurol 201:32-48.
Fernandes KJL, McKenzie IA, Mill P, Smith KM, Akhavan M, BarnabeHeider F, Biernaskie J, Junek A, Kobayashi NR, Toma JG, Kaplan DR Labosky PA, Rafuse V, Hui C-C, Miller FD (2004) A dermal niche for mutipotent adult skin-derived precursor cells. Nat Cell Biol 6:1082-1093.

Fitch MT, Silver J (1997) Glial cell extracellular matrix: boundaries for axon growth in development and regeneration. Cell Tissue Res 290:379-384.

Gilmore SA, Sims TJ (1993) Patterns of Schwann cell myelination of axons within the spinal cord. J Chem Neuroanat 6:191-199.

Grimpe B, Silver J (2002) The extracellular matrix in axon regeneration. Prog Brain Res 137:333-349.

Guest JD, Rao A, Olson L, Bunge MB, Bunge RP (1997) The ability of human Schwann cell grafts to promote regeneration in the transected nude rat spinal cord. Exp Neurol 148:502-522.

Harel NY, Strittmatter SM (2006) Can regenerating axons recapitulate developmental guidance during recovery from spinal cord injury? Nat Rev Neurosci 7:603-616.

Hargreaves K, Dubner R, Brown F, Flores C, Joris J (1988) A new and sensitive method for measuring thermal nociception in cutaneous hyperglasia. Pain 32:77-88.

Hausmann ON (2003) Post-traumatic inflammation following spinal cord injury. Spinal Cord 41:369-378.

Hill CE, Moon LD, Wood PM, Bunge MB (2006) Labeled Schwann cell transplantation: cell loss, host Schwann cell replacement, and strategies to enhance survival. Glia 53:338-343.

Hofstetter CP, Holmstrom NA, Lilja JA, Schweinhardt P, Hao J, Spenger C, Wiesenfeld-Hallin Z, Kurpad SN, Frisen J, Olson L (2005) Allodynia limits the usefulness of intraspinal neural stem cell grafts; directed differentiation improves outcome. Nat Neurosci 8:346-353.

Jessen KR, Mirsky R (2002) Signals that determine Schwann cell identity. J Anat 200:367-376.

Jessen KR, Mirsky R (2005) The origin and development of glial cells in peripheral nerves. Nat Rev Neurosci 6:671-682.

Joannides A, Gaughwin P, Schwiening C, Majed H, Sterling J, Compston A, Chandran S (2004) Efficient generation of neural precursors from adult human skin: astrocytes promote neurogenesis from skin-derived stem cells. Lancet 364:172-178.

Jones LL, Margolis RU, Tuszynski MH (2003) The chondroitin sulfate proteoglycans neurocan, brevican, phosphacan, and versican are differentially regulated following spinal cord injury. Exp Neurol 182:399-411.

Jones TB, McDaniel EE, Popovich PG (2005) Inflammatory-mediated injury and repair in the traumatically injured spinal cord. Curr Pharm Des 11:1223-1236.

Karimi-Abdolrezaee S, Eftekharpour E, Wang J, Morshead CM, Fehlings MG (2006) Delayed transplantation of adult neural precursor cells promotes remyelination and functional neurological recovery after spinal cord injury. J Neurosci 26:3377-3389.

Keirstead HS, Morgan SV, Wilby MJ, Fawcett JW (1999) Enhanced axonal regeneration following combined demyelination plus Schwann cell transplantation therapy in the injured adult spinal cord. Exp Neurol 159:225-236.

Keirstead HS, Nistor G, Bernal G, Totoiu M, Cloutier F, Sharp K, Steward O (2005) Human embryonic stem cell-derived oligodendrocyte progenitor cell transplants remyelinate and restore locomotion after spinal cord injury. J Neurosci 25:4694-4705.

Kocsis JD, Akiyama Y, Lankford KL, Radtke C (2002) Cell transplantation of peripheral-myelin-forming cells to repair the injured spinal cord. J Rehabil Res Dev 39:287-298.

Liu S, Qu Y, Stewart TJ, Howard MJ, Chakrabortty S, Holekamp TF, McDonald JW (2000) Embryonic stem cells differentiate into oligodendrocytes and myelinate in culture and after spinal cord transplantation. Proc Natl Acad Sci USA 97:6126-6131.

Macias MY, Syring MB, Pizzi MA, Crowe MJ, Alexanian AR, Kurpad SN (2006) Pain with no gain: allodynia following neural stem cell transplantation in spinal cord injury. Exp Neurol 201:335-348.

McKenzie IA, Biernaskie J, Toma JG, Midha R, Miller FD (2006) Skinderived precursors generate myelinating Schwann cells for the injured and dysmyelinated nervous system. J Neurosci 26:6651-6660.

Metz GA, Whishaw IQ (2002) Cortical and subcortical lesions impair skilled walking in the ladder rung walking test: a new task to evaluate foreand hindlimb stepping, placing, and co-ordination. J Neurosci Methods 115:169-179.

Monnier PP, Sierra A, Schwab JM, Henke-Fahle S, Mueller BK (2003) The 
Rho/ROCK pathway mediates neurite growth-inhibitory activity associated with the chondroitin sulfate proteoglycans of the CNS glial scar. Mol Cell Neurosci 22:319-330.

Morshead CM, Reynolds BA, Craig CG, McBurney MW, Staines WA, Morassutti D, Weiss S, van der Kooy D (1994) Neural stem cells in the adult mammalian forebrain: a relatively quiescent subpopulation of subependymal cells. Neuron 13:1071-1082.

Nakamura M, Bregman BS (2001) Differences in neurotrophic factor gene expression profiles between neonate and adult rat spinal cord after injury. Exp Neurol 169:407-415.

Nistor GI, Totoiu MO, Haque N, Carpenter MK, Keirstead HS (2005) Human embryonic stem cells differentiate into oligodendrocytes in high purity and myelinate after spinal cord transplantation. Glia 49:385-396.

Ogawa Y, Sawamoto K, Miyata T, Miyao S, Watanabe M, Nakamura M, Bregman BS, Koike M, Uchiyama Y, Toyama Y, Okano H (2002) Transplantation of in vitro-expanded fetal neural progenitor cells results in neurogenesis and functional recovery after spinal cord contusion injury in adult rats. J Neurosci Res 69:925-933.

Okano H, Ogawa Y, Nakamura M, Kaneko S, Iwanami A, Toyama Y (2003) Transplantation of neural stem cells into the spinal cord after injury. Semin Cell Dev Biol 14:191-198.

Oudega M, Xu XM (2006) Schwann cell transplantation for repair of the adult spinal cord. J Neurotrauma 23:453-467.

Paino CL, Bunge MB (1991) Induction of axon growth into Schwann cell implants grafted into lesioned adult rat spinal cord. Exp Neurol 114:254-257.

Paino CL, Fernandez-Valle C, Bates ML, Bunge MB (1994) Regrowth of axons in lesioned adult rat spinal cord: promotion by implants of cultured Schwann cells. J Neurocytol 23:433-452.

Pearse DD, Barakat DJ (2006) Cellular repair strategies for spinal cord injury. Expert Opin Biol Ther 6:639-652.

Pearse DD, Pereira FC, Marcillo AE, Bates ML, Berrocal YA, Filbin MT, Bunge MB (2004a) cAMP and Schwann cells promote axonal growth and functional recovery after spinal cord injury. Nat Med 10:610-616.

Pearse DD, Marcillo AE, Oudega M, Lynch MP, Wood PM, Bunge MB (2004b) Transplantation of Schwann cells and olfactory ensheathing glia after spinal cord injury: does pretreatment with methylprednisolone and interleukin-10 enhance recovery? J Neurotrauma 21:1223-1239.

Pinzon A, Calancie B, Oudega M, Noga BR (2001) Conduction of impulses by axons regenerated in a Schwann cell graft in the transected adult rat thoracic spinal cord. J Neurosci Res 64:533-541.

Ramer LM, Au E, Richter MW, Liu J, Tetzlaff W, Roskams AJ (2004a) Peripheral olfactory ensheathing cells reduce scar and cavity formation and promote regeneration after spinal cord injury. J Comp Neurol 473:1-15.

Ramer LM, Richter MW, Roskams AJ, Tetzlaff W, Ramer MS (2004b) Peripherally-derived olfactory ensheathing cells do not promote primary afferent regeneration following dorsal root injury. Glia 47:189-206.

Rasband MN, Trimmer JS (2001a) Subunit composition and novel localization of $\mathrm{K}^{+}$channels in spinal cord. J Comp Neurol 429:166-176.

Rasband MN, Trimmer JS (2001b) Developmental clustering of ion channels at and near the node of Ranvier. Dev Biol 236:5-16.

Rasband MN, Trimmer JS, Schwarz TL, Levinson SR, Ellisman MH, Schachner M, Shrager P (1998) Potassium channel distribution, clustering, and function in remyelinating rat axons. J Neurosci 18:36-47.

Reynolds BA, Weiss S (1992) Generation of neurons and astrocytes from isolated cells of the adult mammalian central nervous system. Science 255:1707-1710.

Reynolds BA, Tetzlaff W, Weiss S (1992) A multipotent EGF-responsive striatal embryonic progenitor cell produces neurons and astrocytes. J Neurosci 12:4565-4574.

Richardson PM, McGuinness UM, Aguayo AJ (1980) Axons from CNS neurons regenerate into PNS grafts. Nature 284:264-265.

Roy NS, Cleren C, Singh SK, Yang L, Beal MF, Goldman SA (2006) Functional engraftment of human ES cell-derived dopaminergic neurons enriched by coculture with telomerase-immortalized midbrain astrocytes. Nat Med 12:1259-1268.

Schwab JM, Brechtel K, Mueller CA, Failli V, Kaps HP, Tuli SK, Schluesener HJ (2006) Experimental strategies to promote spinal cord regeneration-an integrative perspective. Prog Neurobiol 78:91-116.

Tang X, Davies JE, Davies SJ (2003) Changes in distribution, cell associations, and protein expression levels of NG2, neurocan, phosphacan, brevican, versican $\mathrm{V} 2$, and tenascin-C during acute to chronic maturation of spinal cord scar tissue. J Neurosci Res 71:427-444.

Toma JG, Ahkavan M, Fernandes KJL, Barnabe-Heider F, Sadikot A, Kaplan DR, Miller FD (2001) Isolation of multipotent adult stem cells from the dermis of mammalian skin. Nat Cell Biol 3:778-784.

Toma JG, McKenzie IA, Bagli D, Miller FD (2005) Isolation and characterization of multipotent skin-derived precursors from human skin. Stem Cells 23:727-737.

Tuszynski MH, Weidner N, McCormack M, Miller I, Powell H, Conner J (1998) Grafts of genetically modified Schwann cells to the spinal cord: survival, axon growth, and myelination. Cell Transplant 7:187-196.

Vroemen M, Aigner L, Winkler J, Weidner N (2003) Adult neural progenitor cell grafts survive after acute spinal cord injury and integrate along axonal pathways. Eur J Neurosci 18:743-751.

Wanner IB, Guerra NK, Mahoney J, Kumar A, Wood PM, Mirsky R, Jessen KR (2006) Role of N-cadherin in Schwann cell precursors of growing nerves. Glia 54:439-459.

Watanabe K, Nakamura M, Iwanami A, Fujita Y, Kanemura Y, Toyama Y, Okano H (2004) Comparison between fetal spinal-cord- and forebrainderived neural stem/progenitor cells as a source of transplantation for spinal cord injury. Dev Neurosci 26:275-287.

Waxman SG, Utzschneider DA, Kocsis JD (1994) Enhancement of action potential conduction following demyelination: experimental approaches to restoration of function in multiple sclerosis and spinal cord injury. Prog Brain Res 100:233-243.

Weidner N, Blesch A, Grill RJ, Tuszynski MH (1999) Nerve growth factorhypersecreting Schwann cell grafts augment and guide spinal cord axonal growth and remyelinate central nervous system axons in a phenotypically appropriate manner that correlates with expression of L1. J Comp Neurol 413:495-506.

Widenfalk J, Lundstromer K, Jubran M, Brene S, Olson L (2001) Neurotrophic factors and receptors in the immature and adult spinal cord after mechanical injury or kainic acid. J Neurosci 21:3457-3475.

Xu XM, Guenard V, Kleitman N, Bunge MB (1995) Axonal regeneration into Schwann cell-seeded guidance channels grafted into transected adult rat spinal cord. J Comp Neurol 351:145-160.

Xu XM, Chen A, Guenard V, Kleitman N, Bunge MB (1997) Bridging Schwann cell transplants promote axonal regeneration from both the rostral and caudal stumps of transected adult rat spinal cord. J Neurocytol 26:1-16.

Xu XM, Zhang SX, Li H, Aebischer P, Bunge MB (1999) Regrowth of axons into the distal spinal cord through a Schwann-cell-seeded mini-channel implanted into hemisected adult rat spinal cord. Eur J Neurosci 11:1723-1740.

Yiu G, He Z (2006) Glial inhibition of CNS axon regeneration. Nat Rev Neurosci 7:617-627. 